NBSIR 78-1534

\title{
Monte Carlo Studies of Electron and Photon Transport at Energies up to $1000 \mathrm{MeV}$
}

Stephen M. Seltzer

and

Martin J. Berger

Radiation Physics Division Center for Radiation Research National Bureau of Standards Washington, D.C. 20234

July 1978

Prepared for

Office of Naval Research Arlington, Virginia 22217

$P Q C$

100

. 456

$78-1534$ 

MONTE CARLO STUDIES OF ELECTRON

AND PHOTON TRANSPORT AT

ENERGIES UP TO $1000 \mathrm{MeV}$

Stephen M. Seltzer

and

Martin J. Berger

Radiation Physics Division

Center for Radiation Research

National Bureau of Standards

Washington, D.C. 20234

July 1978

Prepared for

Office of Naval Research

Arlington, Virginia 22217

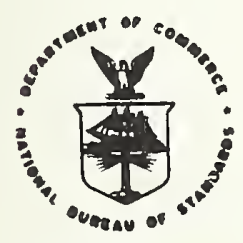

U.S. DEPARTMENT OF COMMERCE, Juanita M. Kieps, Secrotary

Dr. Sidney Harman, Under Secretery

Jordan J. Baruch, Assistant Socrotary for Science and Technology

NATIONAL BUREAU OF STANDARDS, Ernest Ambler, Director 


\title{
MONTE CARLO STUDIES OF ELECTRON AND PHOTON TRANSPORT \\ AT ENERGIES UP TO $1000 \mathrm{MeV}$
}

\author{
Stephen M. Seltzer \\ and \\ Martin J. Berger \\ Radiation Physics Division \\ Center for Radiation Research \\ National Bureau of Standards \\ Washington, DC 20234
}

This report describes caiculations of electron-photon showers initiated by electron beams with energies from 50 to $1000 \mathrm{MeV}$. Results obtained with the Monte Carlo program ETRAN are presented for diverse problems including: (1) the spatial distribution of energy deposited in water, air and lead targets; (2) the escape of scattered electrons and secondary bremsstrahlung from air and lead targets; (3) energy-loss straggling of primary electrons in air; (4) depth-dependent electron spectra (differential tracklength distributions) in air. 


\section{INTRODUCTION}

This report describes calculations of electron-photon showers initiated by electron beams with energies from 50 to $1000 \mathrm{MeV}$ in water, air and lead media. The calculations were done with the Monte Carlo computer program ETRAN.

The ETRAN program was originally developed ' solely as a tool for solving electron transport problems, to be applied at energies up to a few MeV. ${ }^{2}$ The treatment of electron transport in ETRAN is based on a segment model in which the multiple-scattering deflections and energy-losses in successive track segments are sampled from pertinent probability distributions given by analytical theories. The ETRAN program was later extended to include the propagation of secondary bremsstrahlung photons, and was applied in the energy region from a few MeV to $100 \mathrm{MeV}$ where the bremsstrahlung photons are making an increasingly significant contribution to energy transport. 3,4,5 The transport of photons is treated in ETRAN by simulating photoelectric absorption, Compton scattering and pair production in rather direct analogy to the physical processes.

In the present work the application of the ETRAN code has been extended to the energy region from 100 to $1000 \mathrm{MeV}$, where energy transport by photons is the dominant phenomenon and energy transport by electrons is a significant but relatively minor factor.

Because of its origin as an electron transport program, ETRAN treats energy losses and angular deflections in successive track segments in rather detailed fashion. Energy losses are sampled according to the Landau ${ }^{6}$ energyloss straggling distribution, instead of being evaluated in the continuousslowing-down approximation. Angular multiple-scattering deflections are sampled from the Goudsmit-Saunderson ${ }^{7}$ distribution, and the latter is evaluated with the use of an elastic scattering cross section based on a 
combination of the Mott ${ }^{8}$ cross section with a screening correction due to Moliere. ${ }^{9}$ The Mott cross section is an improvement over the Rutherford cross section, taking into account relativistic and spin effects according to the Dirac equation.

There is a considerable literature on electron-photon shower calculations at high energies. Th early work was largely analytical and is summarized, for example, in the book by Rossi. ${ }^{10}$ Later work has been done mainly with use of the Monte Carlo method, usually with a track-segment model for electron transport. 11-18 The track segment models used in high-energy shower calculations differ from those in ETRAN in two respects: (a) the sampling of angular deflections from a Gaussian diffusion approximation, or from the Molière theory based on the Rutherford cross section; (b) the use of the continuous-slowing-down approximation rather than Landau's straggling theory to take into account the cumulative effect of small energy transfers to atoms. Additional simplifications have sometimes been employed to reduce the computational effort involved in following the large numbers of secondary electrons in a shower. The various Monte Carlo programs are similar to ETRAN in that they carefully simulate events resulting in sudden large energy transfers to knock-on electrons or bremsstrahlung photons whose subsequent histories are also followed.

The Goudsmit-Saunderson multiple scattering distribution used in ETRAN has the form of a Legendre series, which - while formally exact - converges quite slowly at high energies. The use of the more approximate Molière distribution, in which this Legendre series is already implicitly summed, avoids this convergence difficulty. However, it appears difficult to treat the energy history of electrons at very high energies adequately while at the same time choosing track segments long enough so that the available multiple-scattering theories are fully valid. In a refined model one might want to sample from a plural-scattering distribution valid for any number of collisions, but such a 
refinement is probably unwarranted in the context of a high-energy shower calculation. We also note that the use of the Landau straggling distribution in ETRAN complicates the program and lengthens the numerical computations compared to the use of the continuous-slowing-down approximation.

Finally, it should be pointed out that the refinement of the multiplescattering distributions used to simulate electron tracks is only one of the factors determining the length of the entire Monte Carlo calculation. Another, perhaps more important, factor is the number of segments into which each track is divided. The choice of the lengths of successive segments should ideally depend on the electron energy, and should be arranged so as to obtain a faithful model of the electron transport, while at the same time minimizing the number of segments.

In the present exploratory calculations, we were mainly interested in seeing how well the ETRAN code works at very high energies, and in obtaining immediate answers to certain problems. We therefore have made no effort to optimize the choice of track segments (which would require a good deal of numerical experimentation) but instead have made simple choices taken over from earlier work at lower energies. For example, when sampling the showers in water generated by an electron with an initial energy of $1 \mathrm{GeV}$, we have chosen segments such that the average energy loss per segment was approximately $11 \%$ of the electron energy at the beginning of the segment, and have further subdivided each segment into two equal sub-segments for the purpose of sampling multiple-scattering angular deflections. It appears to us now that the segment sizes were somewhat too large at high energies (near the source energy) and too small for the numerous secondary electrons with low energies.

The results to be presented in the following Sections of this Report fall into five categories: (1) spatial distribution of energy deposition (absorbed dose) in water and air, as a function of depth in the medium and radial distance 
from the beam axis; (2) the backscattering of energy, in the form of secondary bremsstrahlung photons, from a semi-infinite air medium; (3) energy-loss straggling of electrons in air (including a comparison of the Monte Carlo results with a semi-analytical calculation); (4) evaluation of electron flux spectra as a function of depth in the medium, for primary as well as secondary electrons; (5) interaction of a high-energy electron beam with a lead target, including the spatial distribution of energy deposited in the target and the escape of radiation energy (transmission, reflection) from the target, for both perpendicular and oblique incidence of the beam onto the target.

\section{SPATIAL DISTRIBUTION OF ENERGY DEPOSITION IN WATER AND AIR}

A monoenergetic, narrowly collimated (point-monodirectional) beam of electrons was assumed to irradiate a semi-infinite medium, and energy deposition was calculated as a function of the depth $z$ in the medium and of the radial distance $\rho$ from the beam axis. Actually the target was assumed to be a cylinder centered on the direction of the incident beam, with a radius of $70 \mathrm{~g} / \mathrm{cm}^{2}$ and a length of $460 \mathrm{~g} / \mathrm{cm}^{2}$. The leakage of energy through the lateral surface and the transmission face of the cylinder amounted to only a fraction of a percent of the incident energy.

Electrons in the shower were accurately followed (according to the detailed Monte Carlo model) down to an energy of $2 \mathrm{MeV}$, and the small amount of energy deposition along their residual tracks below that energy was estimated approximately. Photons were followed down to an energy of $10 \mathrm{keV}$. The contribution to the total amount of energy deposited in the target by photons with energies less than $10 \mathrm{keV}$ was insignificant.

Results for the depth dose, i.e. the energy deposition per unit depth (integrated over a 11 radial distances) are given in $\mathrm{Fig} .1$ for a water medium and various electron-beam energies: 
The size of the sample refers to the number of primary electrons. The total number of electrons in the cascade was many times larger. At $1000 \mathrm{MeV}$ it is possible to make comparison with the experimental data of Crannell et al., 19 and with the results obtained by Nelson ${ }^{20}$ with the Monte Carlo program EGS. ${ }^{17}$ The agreement with experiment is quite good and is well within the combined limits of theoretical and experimental error. The agreement with the EGS results is close for most depths; however, for very large depths the EGS results are somewhat lower than the ETRAN and experimental results. This discrepancy is possibly due to the fact that the cylindrical target assumed in Nelson's calculation had a radius of only $24 \mathrm{~g} / \mathrm{cm}^{2}$ (resulting in a significant lateral escape of energy from the target). Beyond the shower maximum the depth-dose curves, on a semi-logarithmic plot, are almost linear, indicating that the transport process is controlled by the approximately exponential attenuation of the secondary bremsstrahlung. In the region up to the shower maximum the transition curves are governed by the penetration of the primary and secondary knock-on electrons. The energy deposition at the surface of the medium is smaller than that which one would predict using the stopping power (mean collision loss), due to the forward transport of energy by energetic knock-on electrons.

A comparison of depth-dose curves for a $500-\mathrm{MeV}$ beam in water and in air is shown in Fig. 2. The transition curve for air is shifted towards smaller depths compared to that for water, because the mass stopping power is larger in air than in water for electrons of the same energy. This difference of stopping power is the result of the polarization of a dense medium by the electron beam (density effect correction). 
Fig. 3a shows the two-dimensional distribution of energy deposition in water from a 1000-MeV beam, as a function of the depth $z$ and radial distance $\rho$ from the axis of the point-monodirectional incident electron beam. The results shown are averaged over the indicated depth intervals. Also shown for comparison are experimental results of Crannell et al.,,$^{19}$ and Monte Carlo (EGS) results of Nelson. ${ }^{20}$ In Nelson's work, the dependence of the energydeposition distribution on the choice of track segment sizes was explored, and the final results reflect a choice which gives very good agreement with Crannell's experimental data. The agreement among the ETRAN, EGS and experimental results is generally to within $20 \%$ or better, which we consider satisfactory in view of the expected theoretical and experimental uncertainties. Fig. 3b compares two-dimensional energy-deposition distributions in water and in air from a $500-\mathrm{MeV}$ beam. The differences are relatively sma17, generally no greater than $20 \%$. The differences are similar in magnitude to those for the corresponding depth-dose curves in Fig. 2, and have their origin in the differences in the density-effect correction to the stopping power for water and air. However, the statistical fluctuations of the Monte Carlo results obscure the situation.

Tables 1 to 4 give the two-dimensional energy-deposition distributions in water for 1000-, 500-, 250-, and 125-MeV beams. The results are presented in terms of the percentage of the incident energy that is deposited in various depth intervals and radial intervals.

\section{BACKSCATTERING OF PHOTONS FROM AN AIR MEDIUM}

When a semi-infinite air medium is irradiated with high-energy electron beams, the transport of energy is predominantly in the forward direction. The very small amount of backscattered energy is nevertheless of interest for radiological safety considerations. We have investigated this problem for the case of monoenergetic, point-monodirectional beams with energies up to 
$1000 \mathrm{MeV}$, and have studied the emergence of radiation energy from a semiinfinite medium as a function of the distance from the electron beam.

We have found in our calculations that the backscattered radiation energy escaping from the medium consists almost entirely of photons, and that only $0.09 \%$ of the energy from a $100-\mathrm{MeV}$ and $0.014 \%$ of the energy from a $1000-\mathrm{MeV}$ beam is backscattered. Such results cannot readily be obtained merely by the direct-analogue simulation of particle trajectories, because excessively large numbers of tracks would have to be sampled. A collision density method has been found effective in dealing with this problem. When generating photon Monte Carlo histories, we focussed attention on the collision density, i.e. the set of all events in which a photon was born or scattered. For each event we then computed (a) the probability that it would emerge from the medium without undergoing a further scattering, and (b) the radial distance between the point of emergence and the beam axis.

An average over the collision density then provides the desired backscattering results. Calculations were done for the following cases:

$\begin{array}{lccccr}\text { Beam energy (MeV) } & 1000 & 500 & 200 & 100 & 50 \\ \begin{array}{c}\text { No. of primary electrons } \\ \text { in Monte Carlo sample }\end{array} & 1000 & 2000 & 2000 & 5000 & 5000\end{array}$

The number of collision points which contributed to the backscattering results amounted to several hundred thousand.

In Fig. 4a, pertaining to a 500-MeV beam, the calculated dose shown is that which would be recorded by an air-equivalent detector placed at the entrance plane of the air medium. The dose is given as a function of the distance $\rho$ from the detector to the beam axis. It has been found that this radial dependence, for a 11 beam energies considered, can be fitted by the empirical one-parameter formula 


$$
D(\rho)=8.1 \times 10^{-5} \rho_{0}\left(e^{-\rho / \rho_{0}}+e^{-4 \rho / \rho_{0}}\right),
$$

where $D(\rho)$ is in MeV/g(air) and the parameter $\rho_{0}$ is in meters. The dependence of $\rho_{0}$ on the beam energy is shown in Fig. $4 b$. The number of backscattered photons, and the amount of backscattered photon energy per $\mathrm{cm}^{2}$ are given in Fig. $4 c$, both as functions of the radial distance $\rho$. Also shown is the mean energy of the backscattered photon, which is independent of radial position. In fact, the mean energy for all beam energies and radial positions was found to be $\sim 150 \mathrm{keV}$.

\section{ENERGY-LOSS STRAGGLING IN AIR}

The ETRAN program has also been used to calculate the energy-loss straggling in air, i.e. the statistical distribution of the energy losses which primary electrons suffer in the course of penetrating a layer of air. In this work, attention was focussed on the distribution in energy of the transmitted electrons, and not on the energy deposition in the air layer, so that secondary electrons could be disregarded. The calculations were done for initial electron energies of 100 and $1000 \mathrm{MeV}$ and for air layers with thicknesses up to $90 \mathrm{~g} / \mathrm{cm}^{2}$. For layers with thicknesses up to $10 \mathrm{~g} / \mathrm{cm}^{2}$, the differences (due to multiple scattering) between the actual pathlengths traveled and the layer thickness $z$ is negligibly small, and it is possible also to make semianalytical calculations of the straggling distribution which can be compared with the Monte Carlo results.

The energy-loss straggling results from the combined effects of (a) energy losses to atoms and secondary electrons in ionization and excitation events, and (b) the emission of bremsstrahlung quanta. Landau ${ }^{6}$ has treated straggling including only effect (a), using the Rutherford cross section. We have extended his treatment to include also effect (b), using the Bethe-Heitler bremsstrahlung cross section (high-energy approximation, complete screening, formula $3 B S(a)$ in the review article of Koch and Motz ${ }^{21}$ ). In setting up the 
solution, we have followed the formalism of Vavilov, ${ }^{22}$ who - in contrast to Landau - imposed an upper limit on the energy transfer in a single collision. In our case, the limit was set equal to one half the kinetic energy in ionizing collisions, and the total kinetic energy in bremsstrahlung events. These restrictions affect the results very little. In any case, our solution, just as those of Landau and Vavilov, is valid only when large energy losses are very rare, and when the average net energy loss over the pathlength considered is small compared to the initial electron energy.

The resulting energy-loss straggling formula is given in the Appendix, and involves a numerical integral over a rather complicated integrand. This integrand is obtained by analytically inverting the Laplace transform of the straggling distribution. We have found the required numerical integration to be difficult, because the integrand is highly oscillatory. Perhaps it would be more efficient to evaluate the inverse of the Laplace transform of the straggling distribution numerically with use of Fast Fourier Transform techniques.

For different air target thicknesses, the results of the Monte Carlo calculations of energy-loss straggling (based on 20,000 histories of primary electrons for each source energy) are compared in Figs. 5a-d with the predictions of the formula given in the Appendix. This formula appears to fit the Monte Carlo data quite well. We find that the value of the most probable energy loss is very little affected by bremsstrahiung losses, and could therefore be predicted by using the Landau theory alone.

The results of the Monte Carlo calculations for air layers of greater thickness are summarized in Fig. 6, which shows the fractional transmission of electrons with energy losses less than $\Delta_{\max }$ through a layer of thickness. 


\section{DIFFERENTIAL TRACKLENGTH DISTRIBUTIONS FOR ELECTRONS IN AIR}

A complete description of electron-photon showers consists of the specification of electron and photon tracklength distributions that are functions of energy, direction and position. Such data can be obtained from the ETRAN program. By way of example, we present here electron tracklength distributions, that are differential in energy, as functions of the depth in the medium. These results are integrals over all directions and radial positions of electrons.

Results for the case of a 500-MeV electron beam in air are shown in Fig. 7 which illustrates the evolution of the electron energy spectrum with increasing depth. Close to the entrance face of the medium, the spectrum consists almost entirely of primary electrons with energies close to the incident beam energy. At larger depths, the primary electron component is increasingly attenuated and a buildup of a secondary component occurs. Eventually the secondary component of the spectrum is completely predominant and has a shape that is rather independent of depth for spectral energies below $\sim 20 \mathrm{MeV}$. These results are based on a sample of 2000 electron-photon cascades.

\section{INTERACTION OF AN ELECTRON BEAM WITH A LEAD TARGET}

In this Section, we consider the interaction of a $500-\mathrm{MeV}$ electron beam with a target of high atomic number. The beam is assumed to be incident on the flat endface of a lead cylinder. The diameter of the target was kept at 30 radiation lengths, and the length of the cylinder was varied up to a value

of 20 radiation lengths. ${ }^{*}$ As shown in Fig. $8 \mathrm{a}$, the point-monodirectional beam was assumed to be incident at the center of the entrance face either perpendicularly or obliquely at an angle $\theta_{0}=70^{\circ}$ with respect to the cylinder axis.

*The radiation length in lead was taken to be $6.5 \mathrm{~g} / \mathrm{cm}^{2}$. 
Fig. 8a shows the depth-dose curves in a lead target for perpendicular and oblique incidence, obtained from a Monte Carlo sample of 500 electron-photon showers. In Fig. $8 \mathrm{~b}$ the same data are replotted as function of the slant distance measured along the incident-beam direction. In this representation the dependence of the depth-dose curves on the initial beam obliquity $\theta_{0}$ is greatly reduced. Fig. $8 c$ shows the radial distribution of deposited energy in the lead target, at a depth of 3-4 radiation lengths (where the depth-dose peaks). The results in Fig. 8c are for perpendicular incidence.

We have also calculated data on the escape of energy from irradiated lead cylinders through the entrance face (reflection), front face (transmission) and through the lateral surface of the cylinder. Fig. 9a shows the dependence of the energy escape on the length of the cylinder, for the cases of perpendicular incidence and oblique incidence. The energy leaving the cylinder by reflection or through the lateral surface is carried almost entirely by photons. For escape through the front face, this is the case also, except for cylinders thin enough so that the primary electrons have a significant chance to be transmitted.

Figs. 9b, $c$ and $d$ show the energy spectra of the electrons and photons transmitted through the front face and reflected from the entrance face of cylinders with diameters of 30 radiation lengths and lengths of 1,5 and 20 radiation lengths. These results are for perpendicular incidence of a 500-MeV electron beam. The photon spectra include, in addition to bremsstrahlung, annihilation quanta and characteristic $x$-rays. The corresponding angular distributions of the escaping radiations are shown in Figs. $9 e, f$ and $g$. 


\section{APPENDIX: ELECTRON ENERGY-LOSS STRAGGLING FORMULA}

Let $P(\Delta) d \Delta$ be the probability that an electron after traversing a pathlength $s$ has suffered a net energy loss between $\Delta$ and $\Delta+d \Delta$. The probability density $P(\Delta)$ can be shown to be

$$
P(\Delta)=\left(2 e^{k a} / \pi T\right) \int_{0}^{\infty} e^{k f_{1}} \cos \left(y \lambda+k f_{2}\right) d y
$$

where

$$
\begin{aligned}
a= & 1-(x / k)\left[n^{\prime}(\ln 2+\gamma)-t\left(n^{\prime}-n t / 2\right)\right], \\
f_{1}= & -\cos y-y \operatorname{si}(y)-(x / k)\left\{n^{\prime}[\ln y-C i(2 y)]+t[b \sin 2 y /(2 y)\right. \\
& \left.\left.+n t(1-\cos 2 y) /(2 y)^{2}\right]\right\}, \\
f_{2}= & y[\ln y-C i(y)]+\sin y-(x / k)\left\{n^{\prime} \operatorname{si}(2 y)-t\left[\left(n^{\prime}-b\right) \cos 2 y /(2 y)\right.\right. \\
& \left.\left.-n t \sin 2 y /(2 y)^{2}\right]\right\}, \\
\lambda= & \frac{2}{T}\left(\Delta-\bar{\Delta}_{C}\right)-k\left\{\ln 2-\gamma-\left[T^{2} / 8-\mu(2 T+\mu) \ln 2\right] /(T+\mu)^{2}\right\} .
\end{aligned}
$$

Other symbols have the following meanings:

$$
\begin{aligned}
& k=2 \xi / T, \\
& \xi=2 \pi r{ }^{2} N_{a}(Z / A)\left(\mu / B^{2}\right) s, \\
& x=4 a r e^{2} N_{a}(Z+1)(Z / A) s, \\
& n=\ln \left(183 Z^{-1 / 3}\right), \\
& n^{\prime}=(4 n+1 / 3) / 3, \\
& t=T /(T+\mu), \\
& b=n^{\prime}-n t, \\
& \bar{\Delta}_{C}=\left(\frac{d E}{d x}\right) \\
& r=l^{s}, \\
& N_{a}=\text { Aviler's constant }=0.577216 \ldots,
\end{aligned}
$$


$r_{e}=$ classical electron radius $=2.818 \times 10^{-13} \mathrm{~cm}$,

$\mu=$ electron rest mass $=0.511 \mathrm{MeV}$,

$\alpha=1 / 137$,

$S i$ is the sine integral,

$\mathrm{C} i$ is the cosine integral,

$T=$ incident electron kinetic energy,

$\beta=$ electron velocity in units of light velocity,

$\mathrm{s}=$ pathlength, $\mathrm{g} / \mathrm{cm}^{2}$,

$Z=a$ tomic number,

$A=$ atomic weight,

$\left(\frac{d E}{d x}\right)_{\text {col }}=$ collision-loss stopping power, $\mathrm{MeV} \mathrm{cm} \mathrm{cm}^{2} / \mathrm{g}$. 


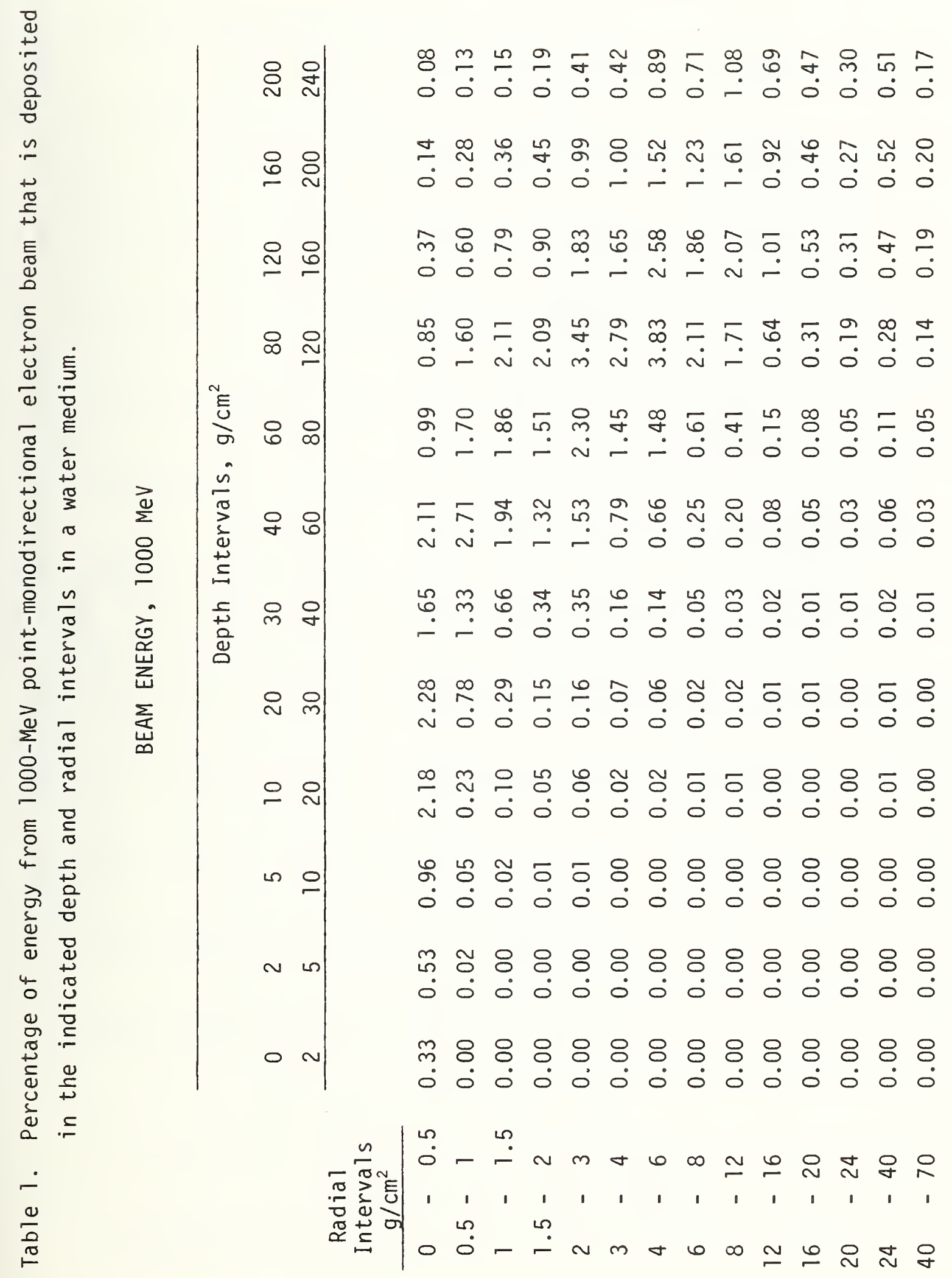




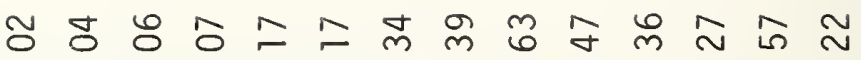

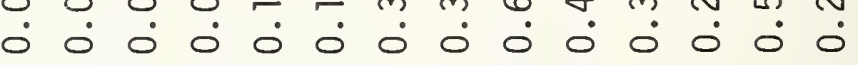

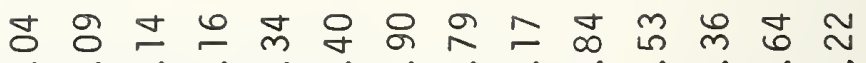

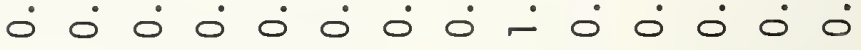

$=\infty \approx$ म $\dot{0} \dot{0} \dot{0} \dot{0} \dot{0}-\dot{\sim} \sim \dot{0} \dot{0} \dot{0}$

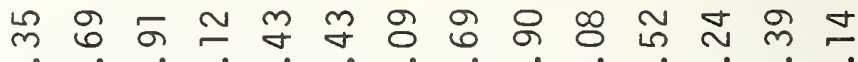
○ $\dot{0} \dot{\sim} \sim \dot{\sim} \dot{\sim} \dot{\sim} \dot{0} \dot{0} \dot{0}$

チ $\dot{0} \dot{0} \dot{\sim} \dot{\sim} \sim \dot{0} \dot{0} \dot{0} \dot{0} \dot{0}$

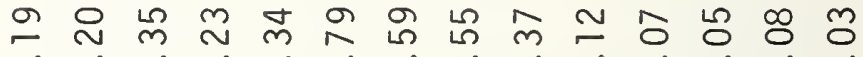

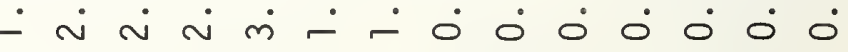

ㅇ

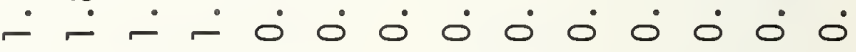

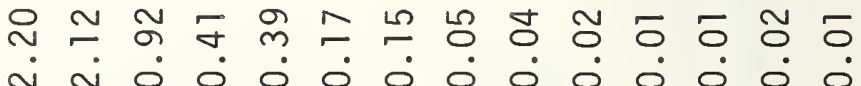
ํ.

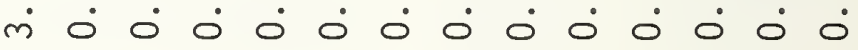

Љ 유

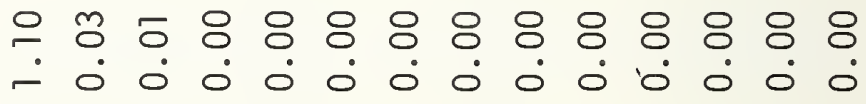

$\begin{array}{llllllllllllll}9 & \circ & 8 & 8 & 8 & 8 & 8 & 8 & 8 & 8 & 8 & 8 & 8 & 8 \\ 0 & 0 & 0 & 0 & 0 & 0 & 0 & 0 & 0 & 0 & 0 & 0 & 0 & 0\end{array}$

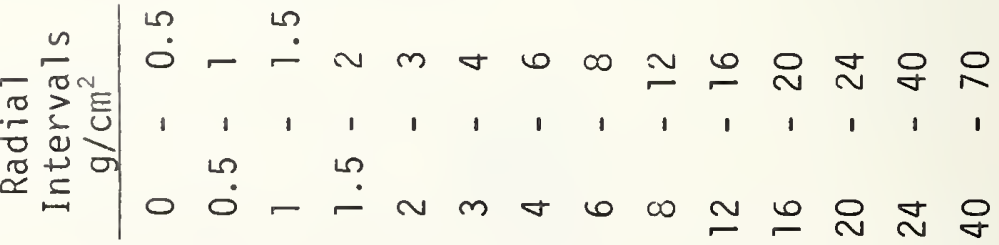




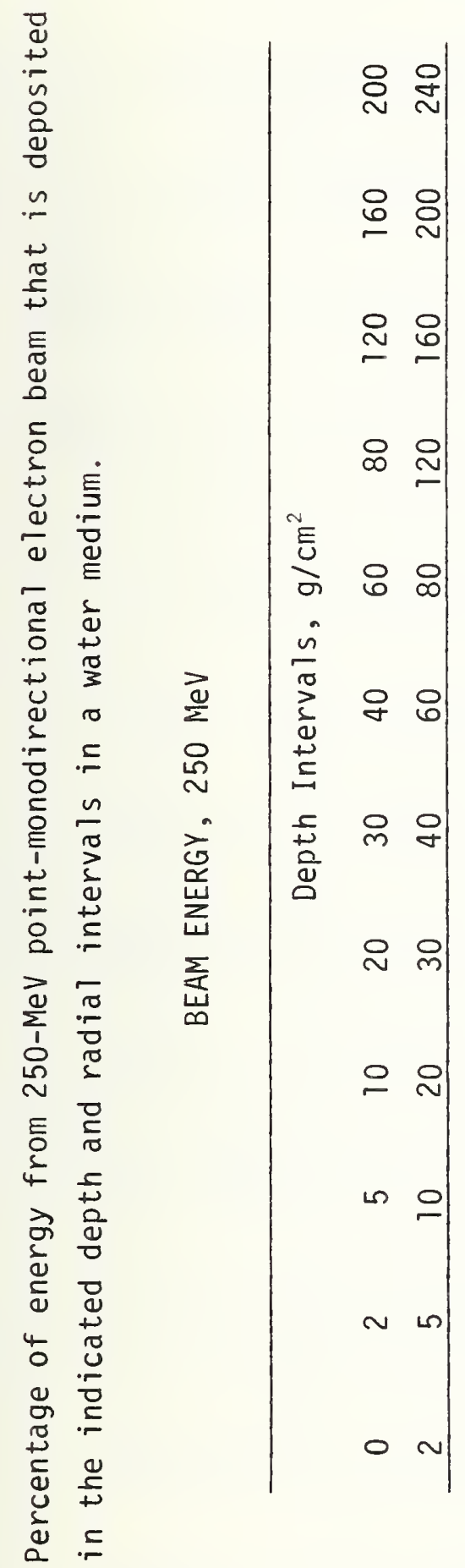

$\frac{m}{\frac{0}{0}}$
ธㅇํㅇㅎㅇ

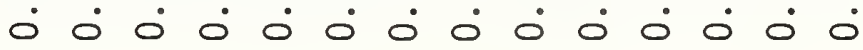

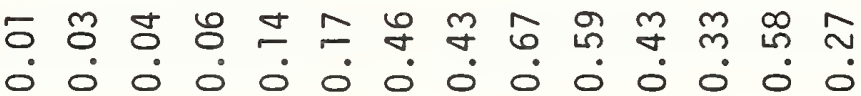

ᄂ

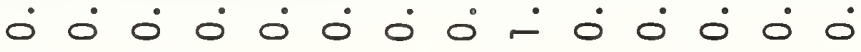

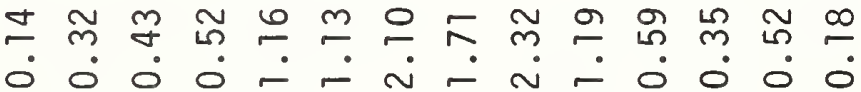

요유ำ

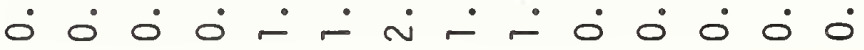

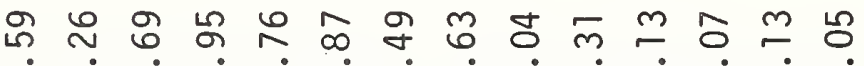

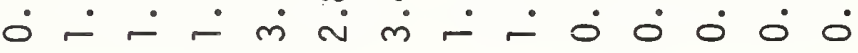

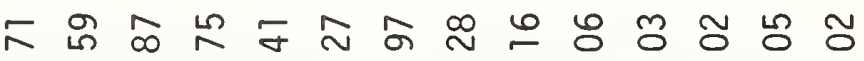

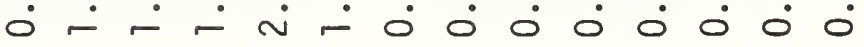

ชิ 으

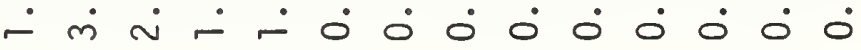

ఇ

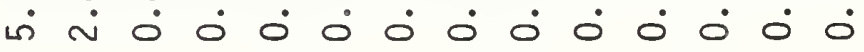

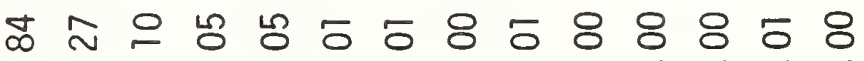

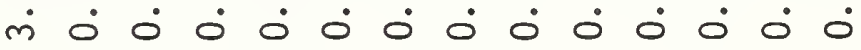

ป

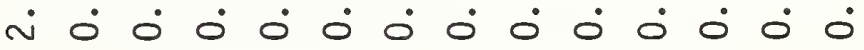

ฆ ㅇ

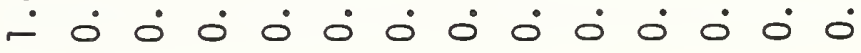

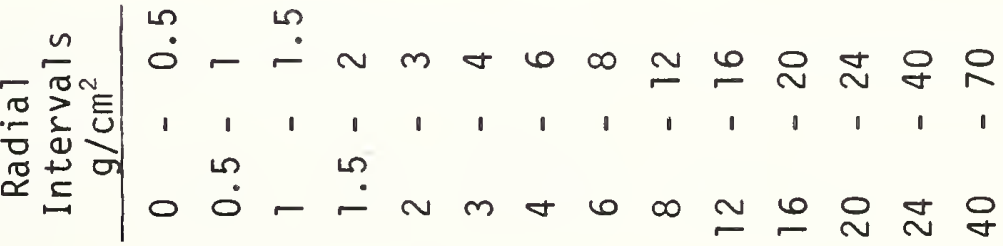




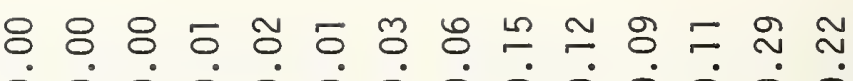

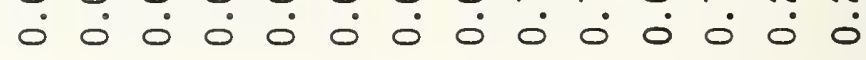

б.

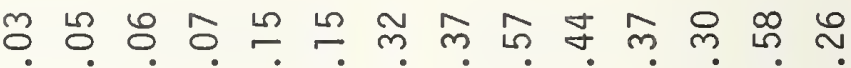

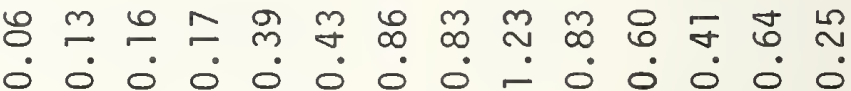

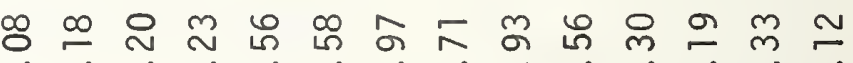

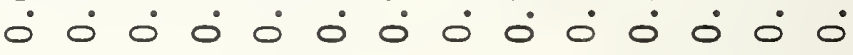

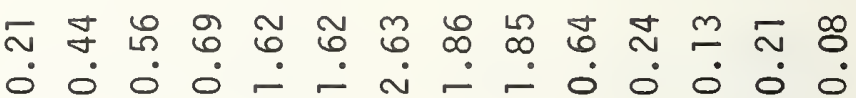

굿ㄴㅇㅇㅛ

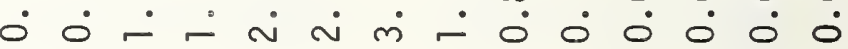

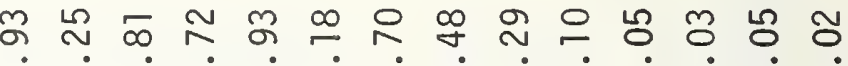

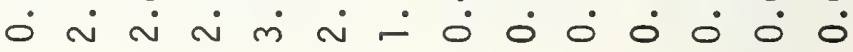

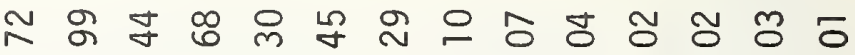

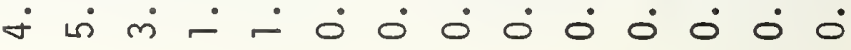

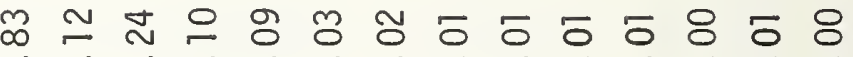

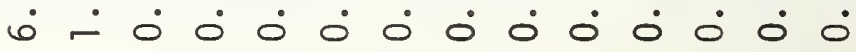

๘

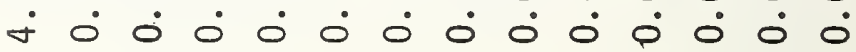

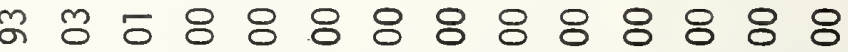

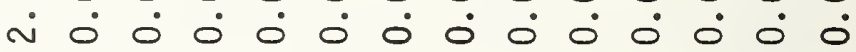

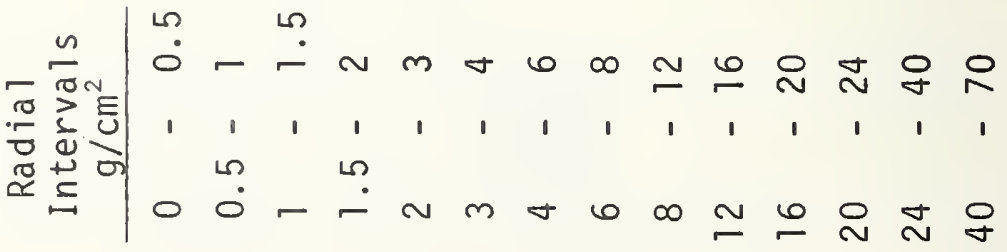


Fig. 1. Energy deposition per unit depth in a water target irradiated by electron beams with energies $T_{\text {g between }} 60$ and $1000 \mathrm{MeV}$. Results are normalized to one incident electron.

The histograms (and the curves in the insert) were calculated with the Monte Carlo code ETRAN. For $\mathrm{T}=1000 \mathrm{MeV}$, the circles (o) and triangles $(\Delta)$ are replicate experimental data of Crannell et al [19]; the crosses $(x)$ are results obtained by Nelson [20] with the Monte Carlo code EGS [17] and have been plotted at the midpoints of his histogram bins.

Fig. 2. Comparison of calculated energy deposition per unit depth in water and air at $500 \mathrm{MeV}$.

Fig. 3. Radial dependence of energy deposition per unit volume from pointmonodirectional electron beam. The electrons are incident along the $z$-axis, and the radial dependence is given as a function of the distance $\rho$ from the $z$-axis. The results are normalized to one incident electron and are averaged over various depth intervals. Some histograms are shifted along the abscissa, with the $\rho=0$ position indicated.

a) 1000-MeV electron beam, water target. Solid histograms are calculated with ETRAN code, crosses $(x)$ are calculated with the EGS code [20], and the dotted histograms are experimental results of Crannel et al [19].

b) 500-MeV electron beam, comparison of energy deposition in water and in air targets. Results were obtained with ETRAN code, assuming a density of $0.001205 \mathrm{~g} / \mathrm{cm}^{3}$ for air. The results for air are shown in scaled form and must be multiplied by the cube of the density to obtain the energy deposition in $\mathrm{MeV} / \mathrm{cm}^{3}$.

Fig. 4. Backscattering of secondary bremsstrahlung photons from an air target irradiated by a point-monodirectional 500-MeV electron beam incident perpendicularly. The results are shown as functions of the distance from the beam axis, and are normalized to one incident electron.

a) Absorbed dose delivered to a small air-equivalent detector at the surface of the target. The dotted curve is a fit by a formula given in Fig. 4b. To convert from MeV/g per incident electron to units of rads/coulomb, the ordinate scale should be multiplied by $10^{11}$.

$J_{N}$ : number of photons per $\mathrm{cm}^{2}$ reflected from target. $\mathrm{J}_{\mathrm{E}}:$ amount of photon energy $\left(\mathrm{MeV}\right.$ per $\mathrm{cm}^{2}$ ) reflected
from target

$\bar{E}=J_{E} / J_{N}$ : mean energy of reflected photons, in MeV

b) Formula fitted to the Monte Carlo data for the absorbed dose from reflected photons. 
Fig. 5. Energy-loss straggling of electrons in air. $P(\Delta)$ is the probability that the electrons will suffer an energy loss between $\Delta$ and $\Delta+d \Delta$ when traversing a plane-parallel target of thickness $z$. The area under each distribution shown is equal to the number transmission coefficient.

Monte Carlo results: Histograms are for an incident electron energy $T_{1}=500 \mathrm{MeV}$. Points are for $\mathrm{T}_{0}=1000 \mathrm{MeV}$ and are plotted at midpoints of histogram bins.

Analytical results (for $T_{0}=500 \mathrm{MeV}$ ): Dotted curves are from Landau's theory of collision-loss straggling. Solid curves are from an extension of Landau's theory that includes fluctuations due to bremsstrahlung emission; dashed curve is an extrapolation.
a) Target thickness $z=2 \mathrm{~g} / \mathrm{cm}^{2}$
b) Target thickness $z=5 \mathrm{~g} / \mathrm{cm}^{2}$
c). Target thickness $z=10 \mathrm{~g} / \mathrm{cm}^{2}$
d) Target thickness $\mathrm{z}=20 \mathrm{~g} / \mathrm{cm}^{2}$

Fig. 6. Integral energy-loss straggling curves in air derived from the Monte Carlo results for $P(\Delta)$ at $500 \mathrm{MeV}$. $Q\left(\Delta_{\text {max }}\right)$ is the fraction of the incident electrons transmitted through a layer of thickness $z$ with an energy loss $\Delta \leqslant \Delta_{\max }$.

Fig. 7. Doubly differential electron tracklength distribution per unit spectral energy and depth. Results were calculated with the ETRAN code for a $500-\mathrm{MeV}$ beam of electrons incident perpendicularly on a semi-infinite air medium. The distributions are normalized to one incident electron and are averaged over the indicated depth intervals. The distributions include primary electrons as well as all secondary electrons from ionizing collisions and photon interactions.

Fig. 8. Spatial distribution of energy deposited in a cylindrical lead target by a $500-\mathrm{MeV}$ electron beam. As indicated in the diagram, the beam is incident at the center of the entrance face of the cylinder, at an angle $\theta_{0}$ with respect to the cylinder axis.

Energy deposition has been computed with the ETRAN code as a function of the depth $z$ in the cylinder and the radial distance $\rho$ from the axis. These distances are expressed in units of the radiation length $X_{R}\left(=6.5 \mathrm{~g} / \mathrm{cm}^{2} \mathrm{~Pb}\right)$.

a) Energy deposition per unit depth, for perpendicular incidence $\left(\theta_{0}=0^{\circ}\right)$ and oblique incidence $\left(\theta_{0}=70^{\circ}\right)$.

b) Same data as in Fig. 8a, plotted as function of the scaled depth $z /\left(X_{R} \cos \theta_{0}\right)$ measured along the direction of incidence.

c) Energy deposition per unit mass as a function of the radial distance $\rho$. Results shown are for $\theta_{0}=0^{\circ}$, and are averaged over the depth interval from 3 to 4 radiation lengths. 
Fig. 9. Transmission and reflection of energy by a cylindrical lead target irradiated with a $500-\mathrm{MeV}$ electron beam. The beam is incident at the center of the entrance face, at an angle $\theta_{0}$ with respect to the axis. The escape of energy from the target has been computed with the ETRAN code as a function of the target thickness $z$ (distance from entrance to front face), for a fixed diameter of $195 \mathrm{~g} / \mathrm{cm}^{2}$ (equal to $30 X_{R}$, where $X_{R}$ is the radiation length).

a) The solid curves show the fraction of the incident energy that emerges from the various parts of the cylinder surface in the form of electrons and bremsstrahlung photons. The dotted curves give the transmission in the form of photons only.

b) Spectra of radiations escaping from target. The quantity plotted is the number per unit spectral energy $E$ of photons or electrons transmitted through the front surface or reflected through the entrance surface. Results are normalized to one incident electron.

The quantity $P_{A}$ is the area under the spike associated with the escape of annihilation quanta. Target thickness is 1 radiation length.

c) Similar to b), but for a target thickness of 5 radiation lengths.

d) Similar to b), but for a target thickness of 20 radiation lengths. Electron spectra are very small and are not shown. Spectrum of reflected photons (not shown) is practically the same as that in c).

e) Angular distribution of radiations escaping from target through the front face (transmission) or the entrance face (reflection). The quantity given is the intensity, i.e. the amount of energy per unit solid angle, carried by electrons and photons. The results are normalized to one incident electron.

The exit angle $\theta$ is defined such that both for transmission and reflection the values of $\theta$ go from $0^{\circ}$ (perpendicular emergence) to $90^{\circ}$. Target thickness is 1 radiation length.

f) Similar to e) but for a target thickness of 5 radiation lengths.

g) Similar to e) but for a target thickness of 20 radiation lengths. 


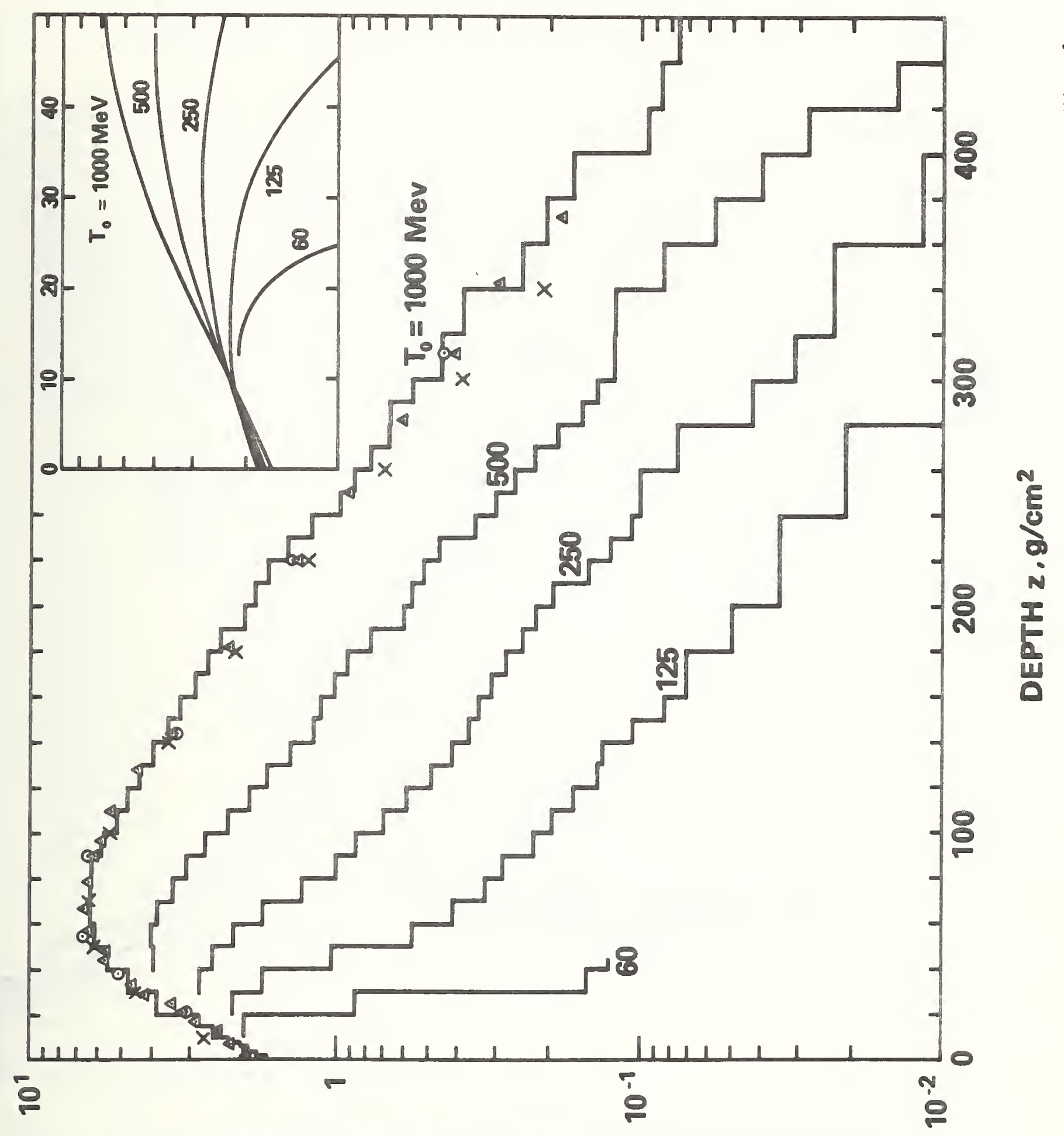

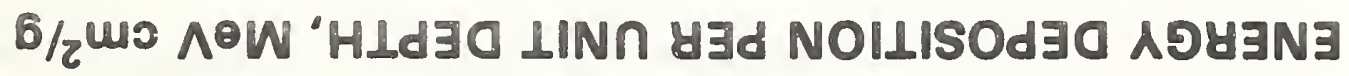




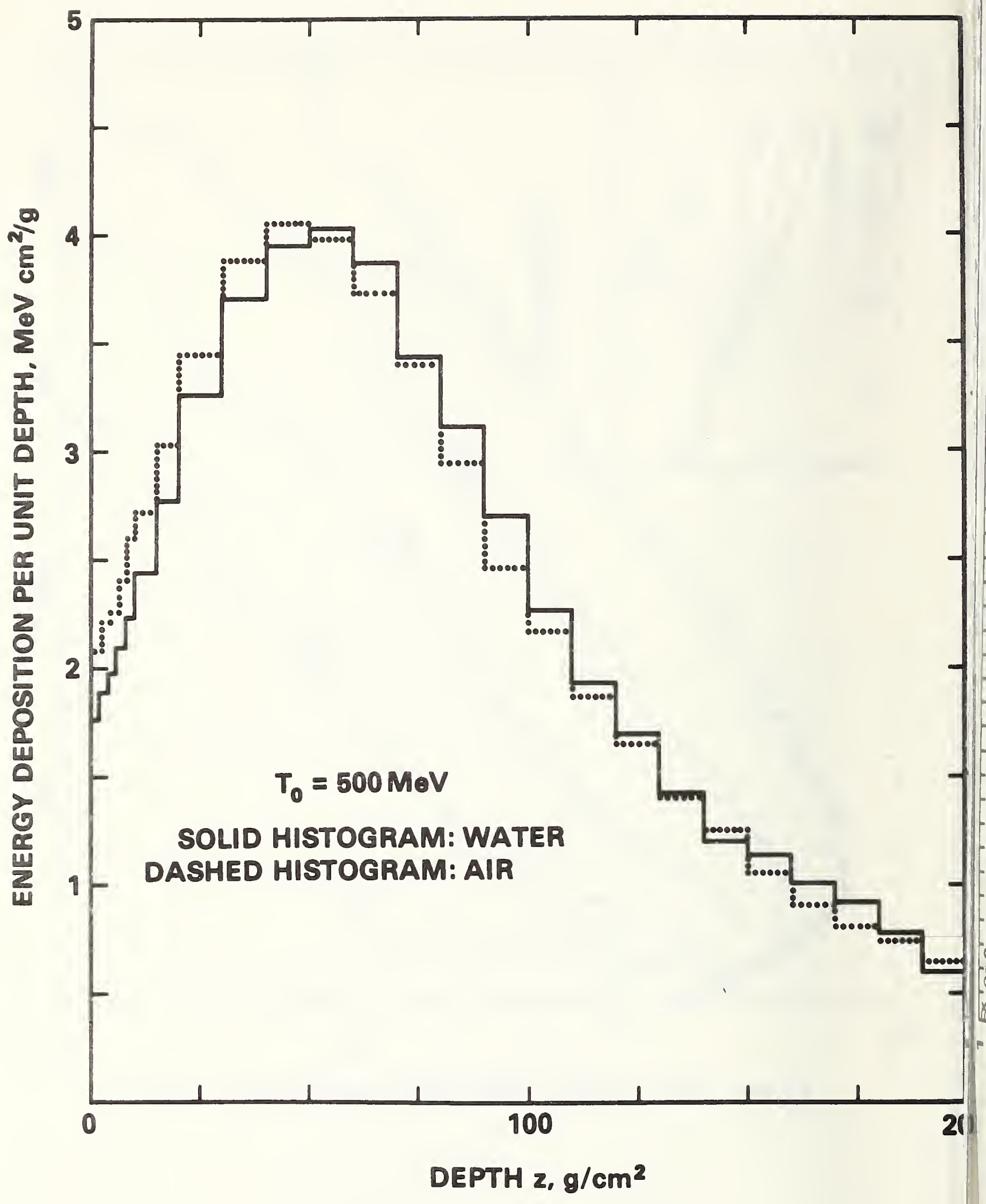

Fig. 2 


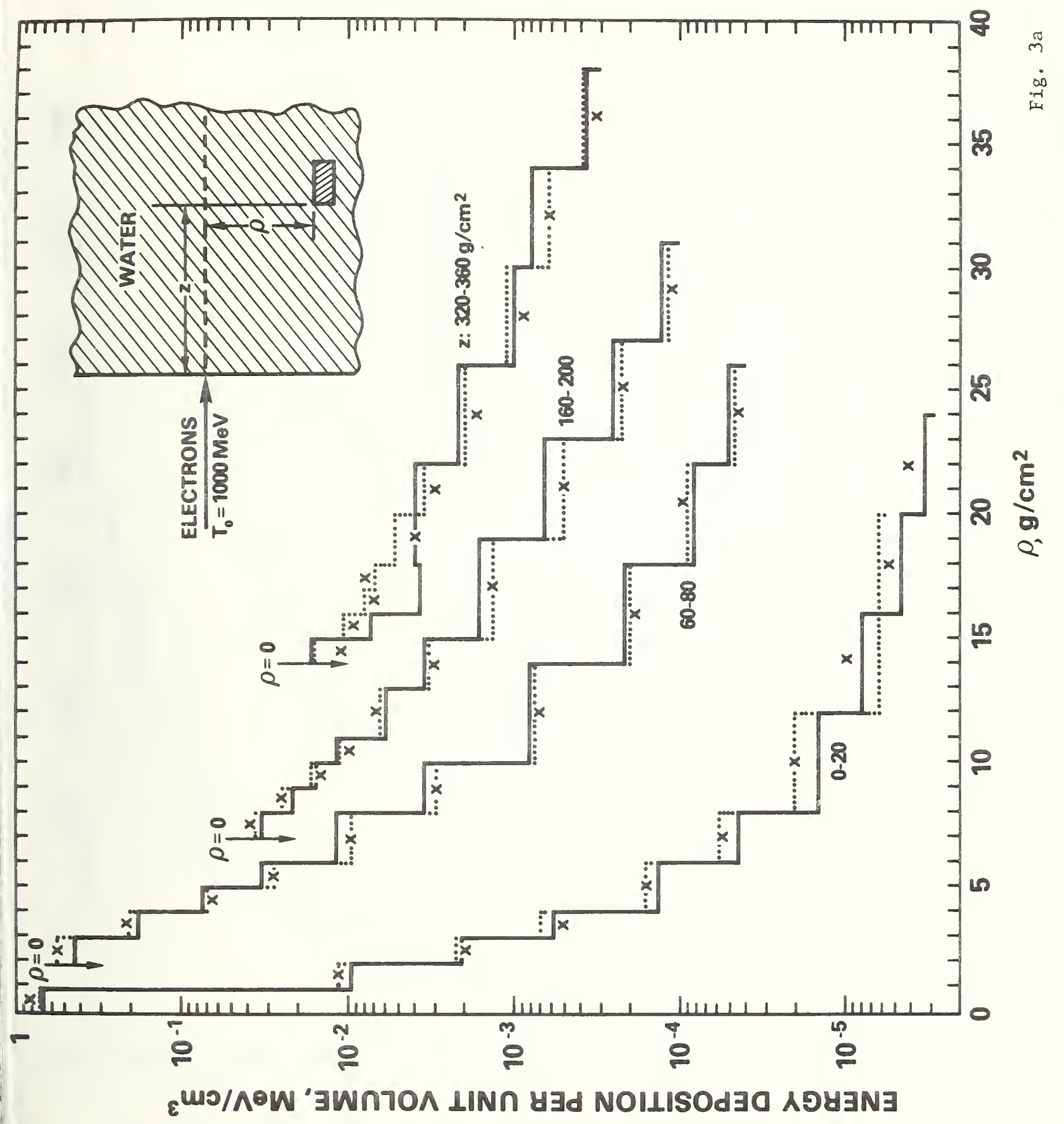




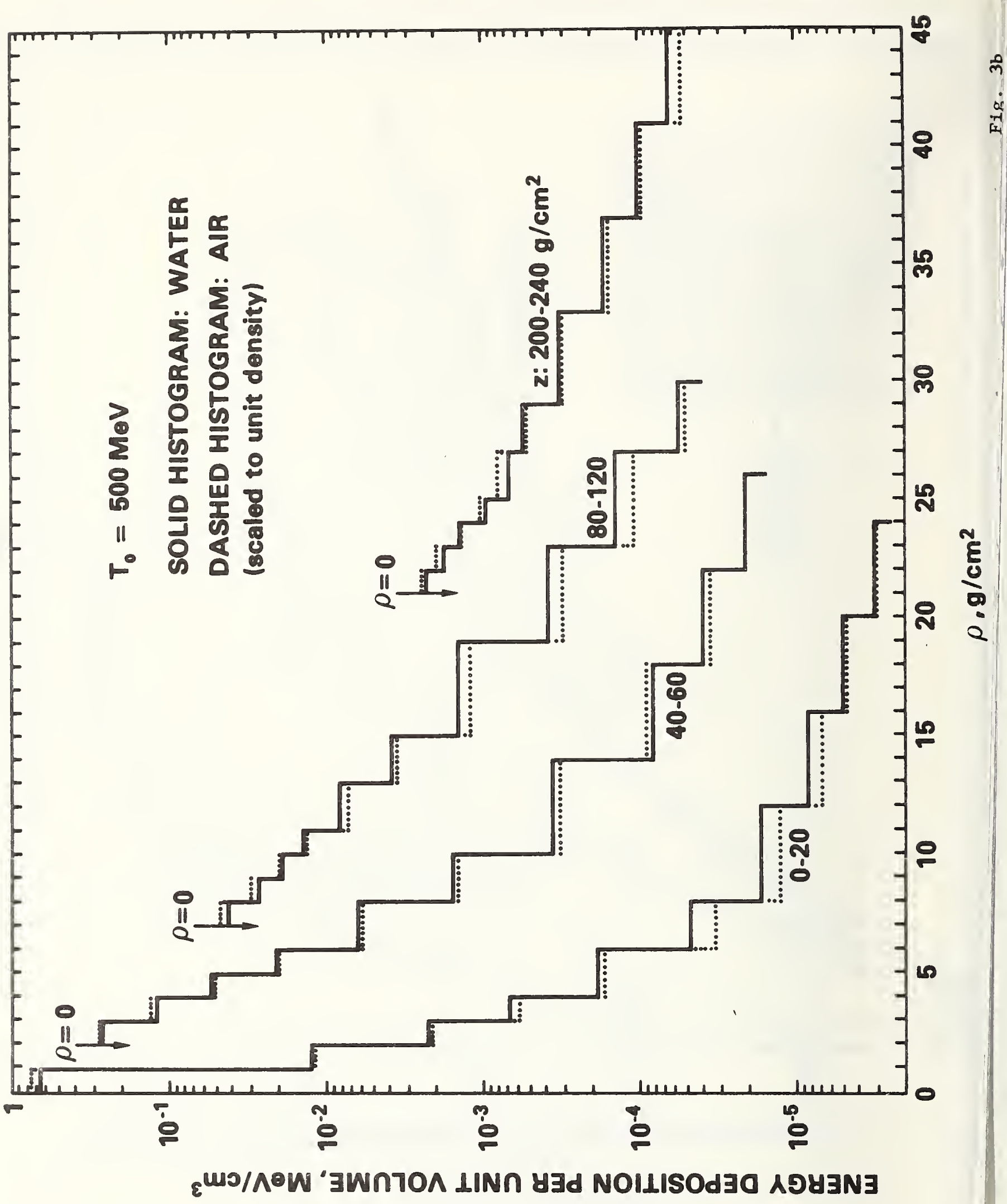




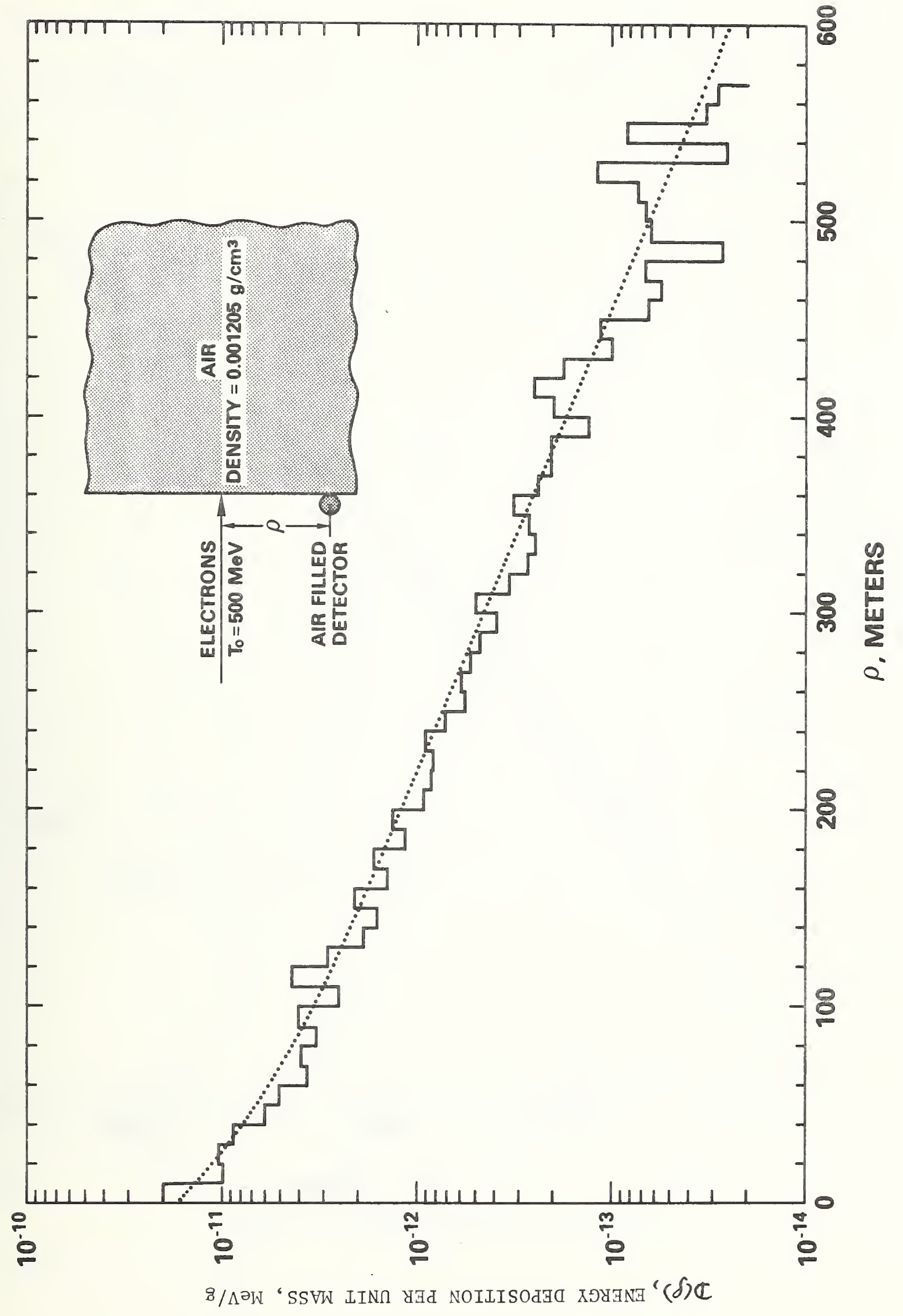




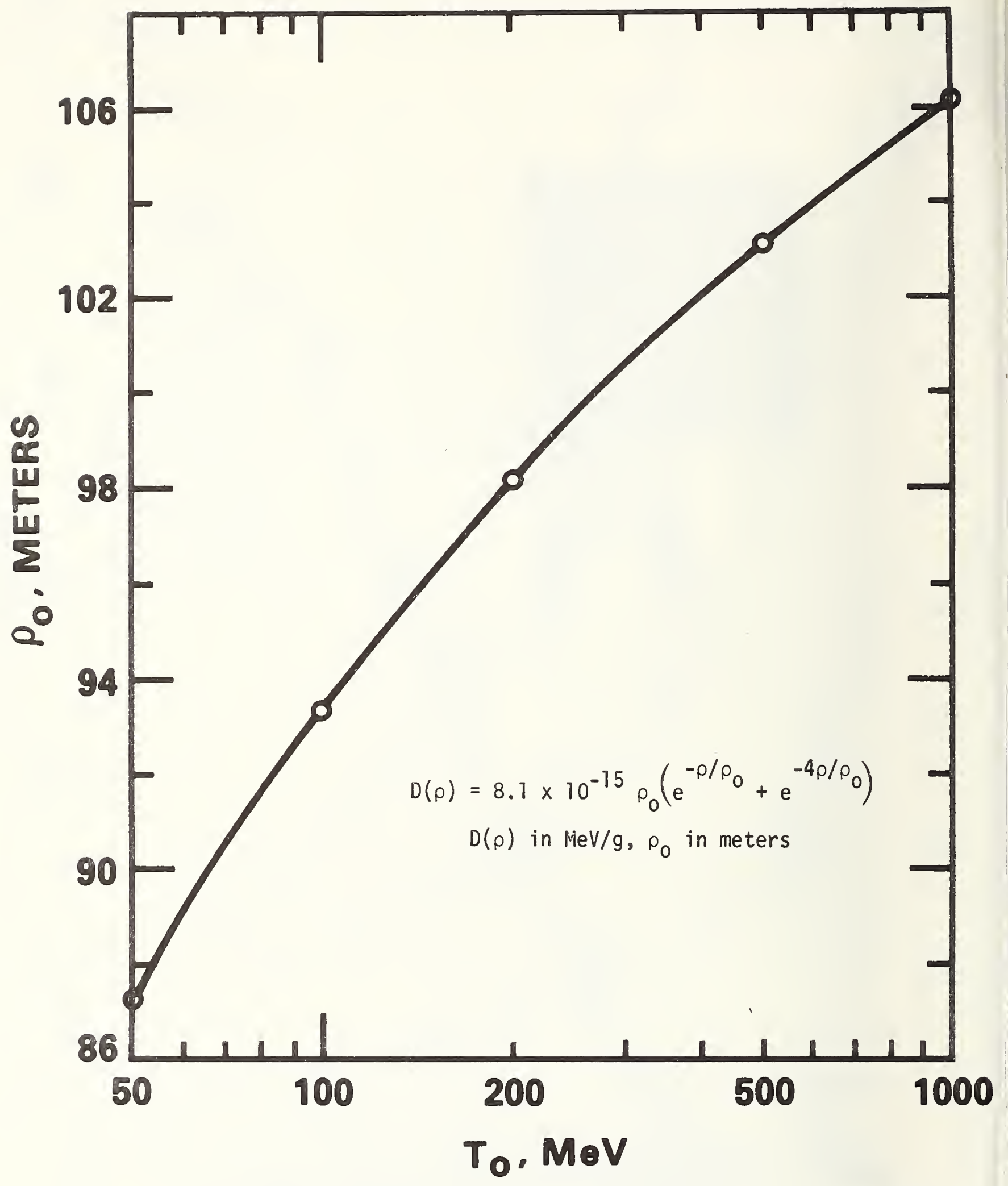

Fig. $4 b$ 
MEAN ENERGY, MOV

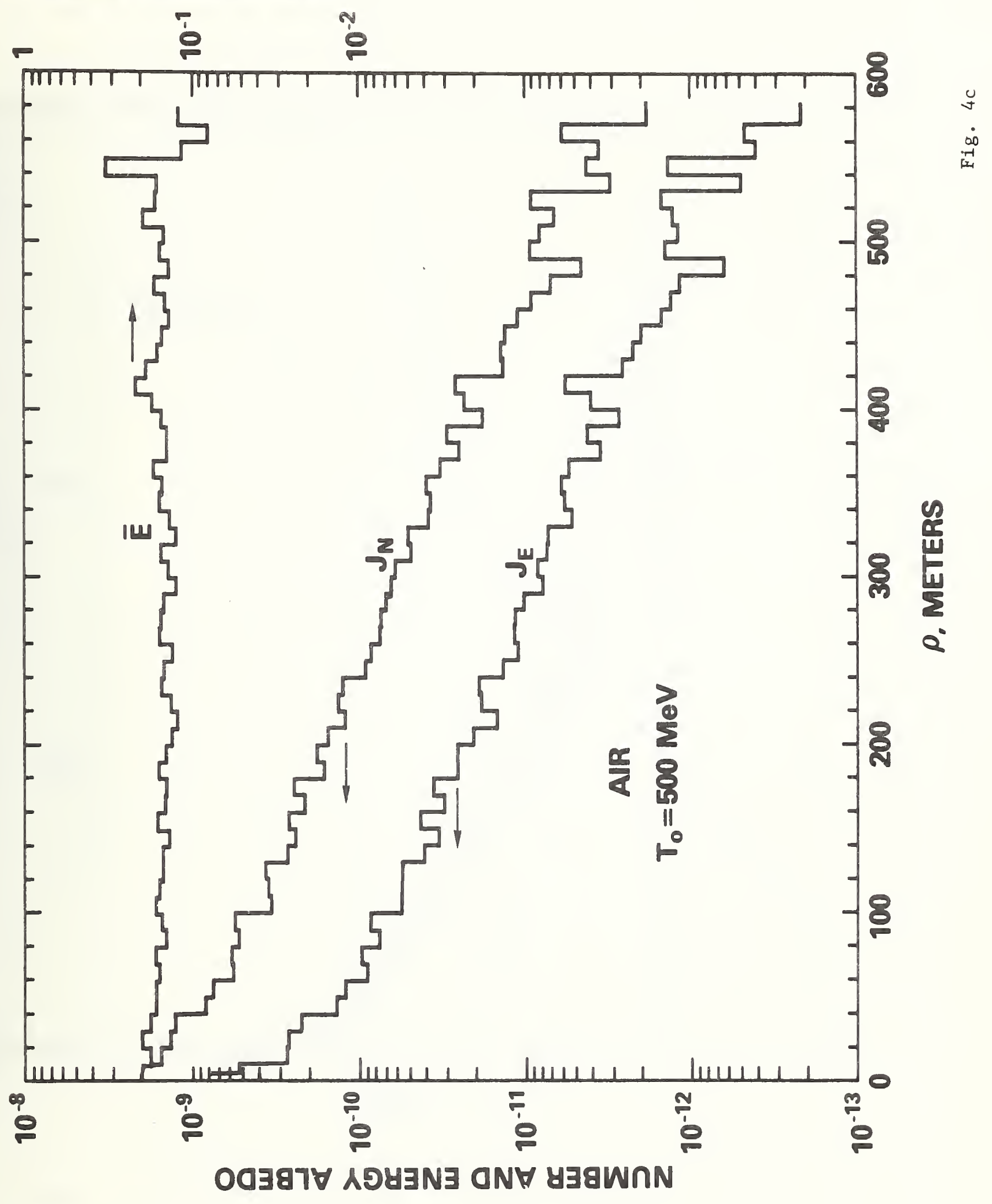




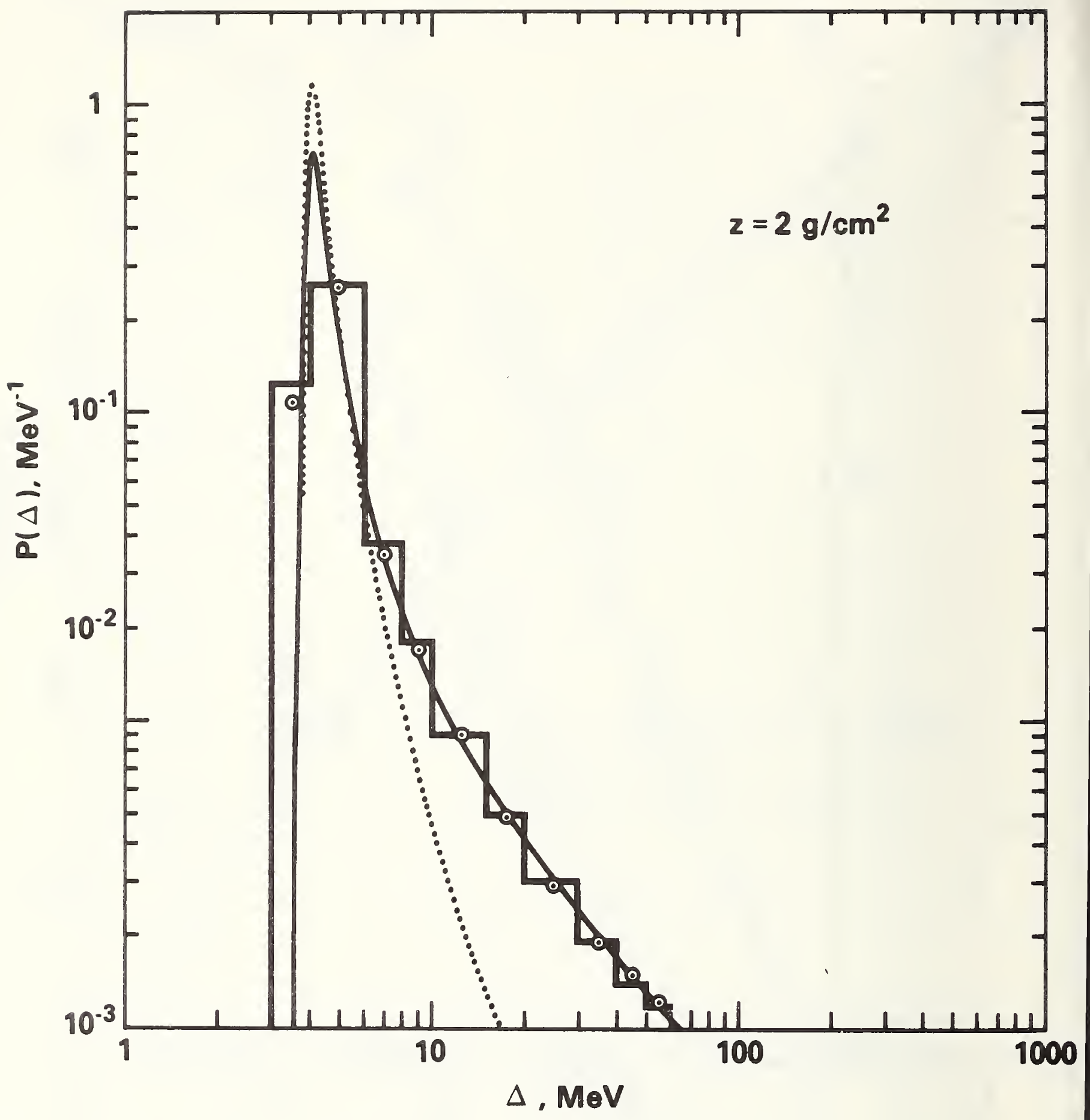

Fig. $5 a$ 


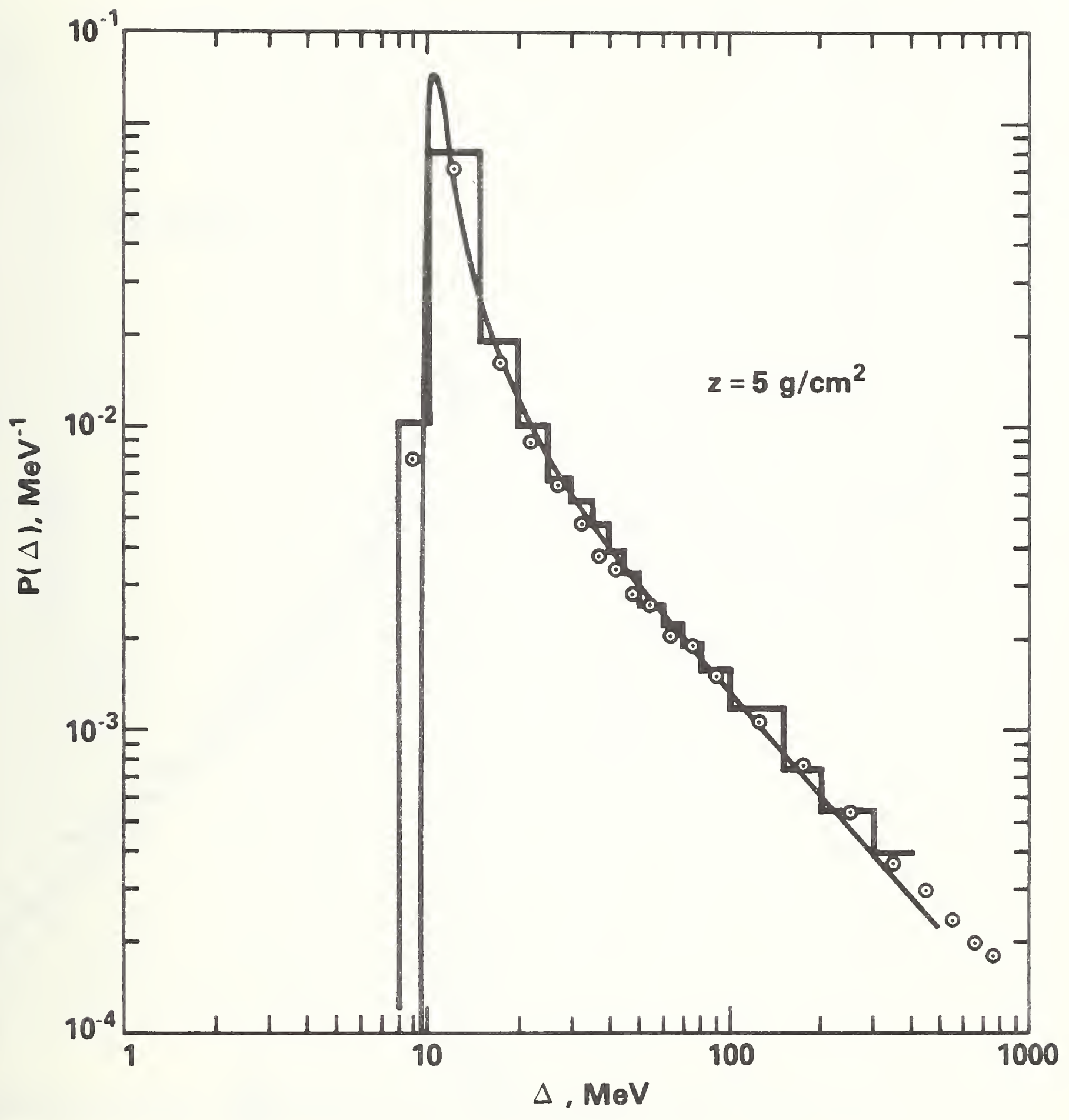

Fig. 5b 


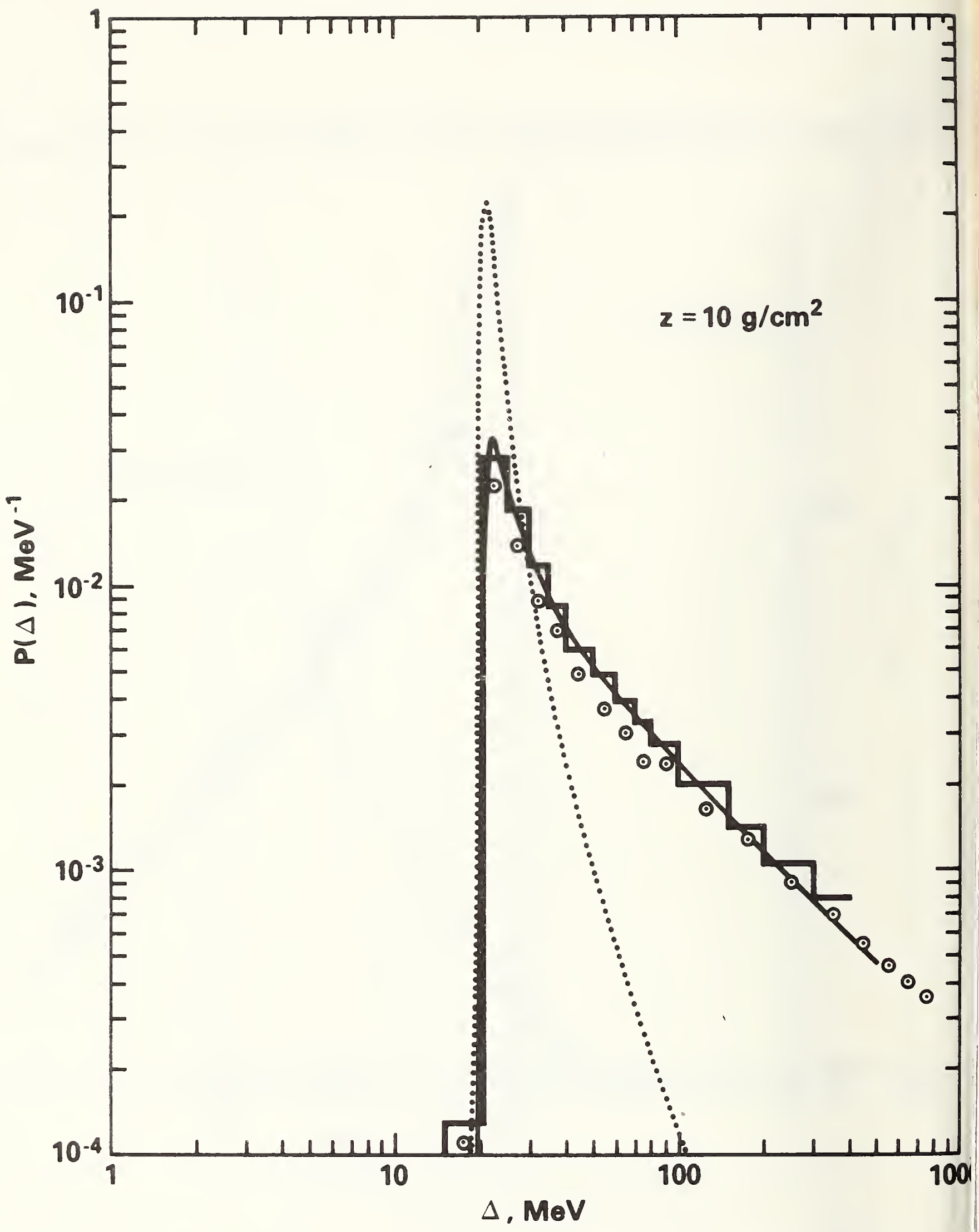

Fig. 5c 


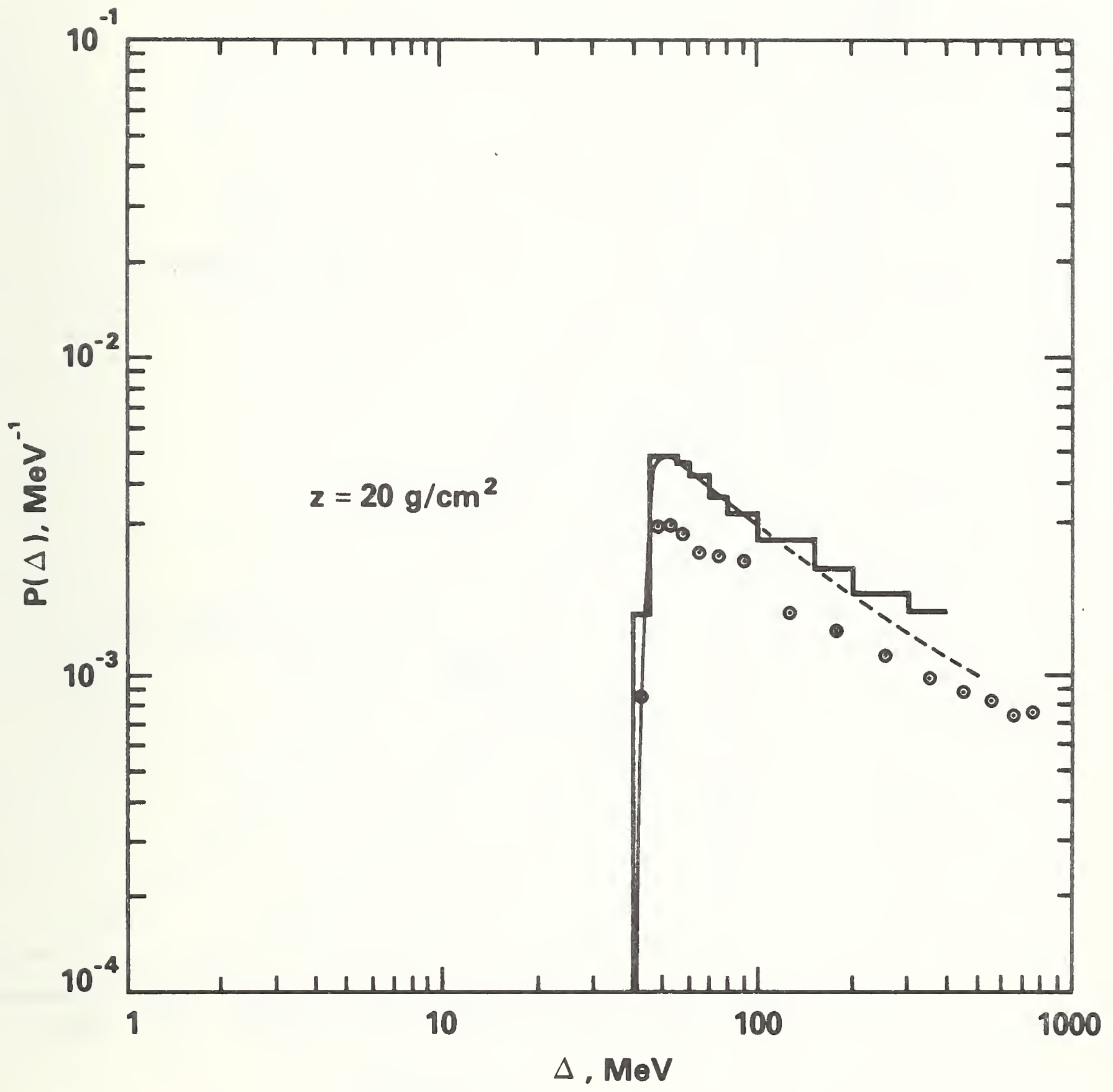

Fig. 5d 


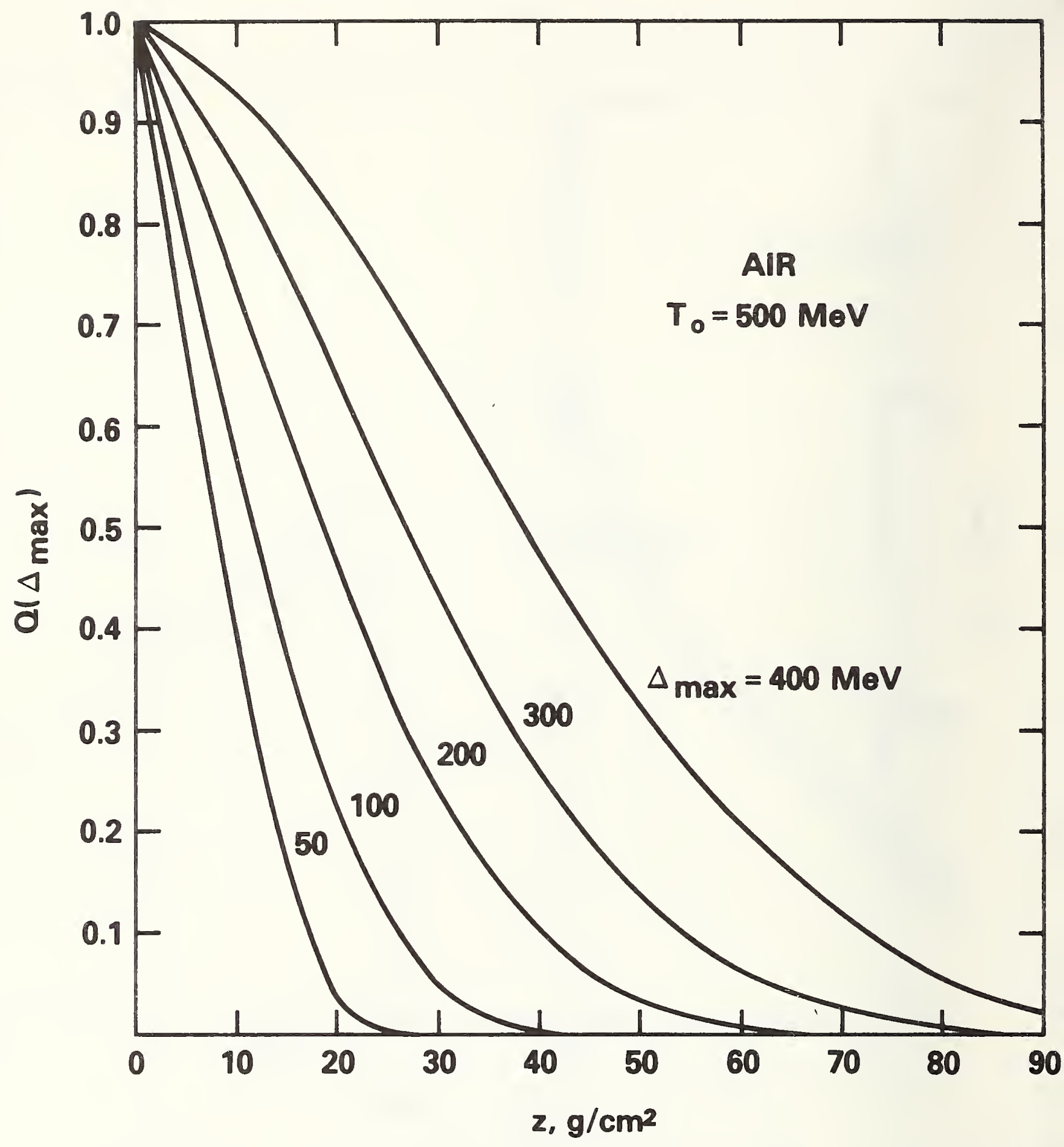

Fig. 6 

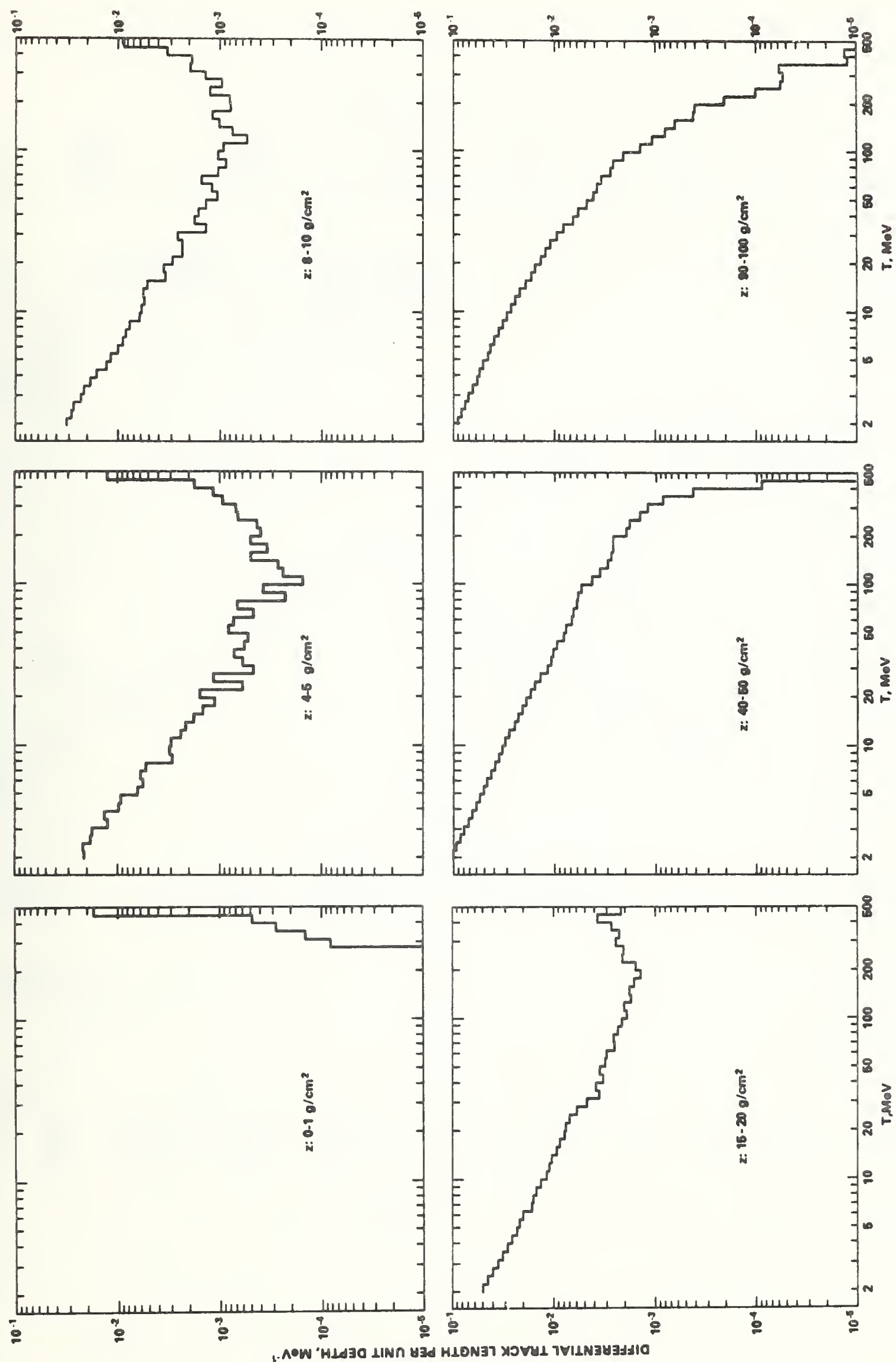


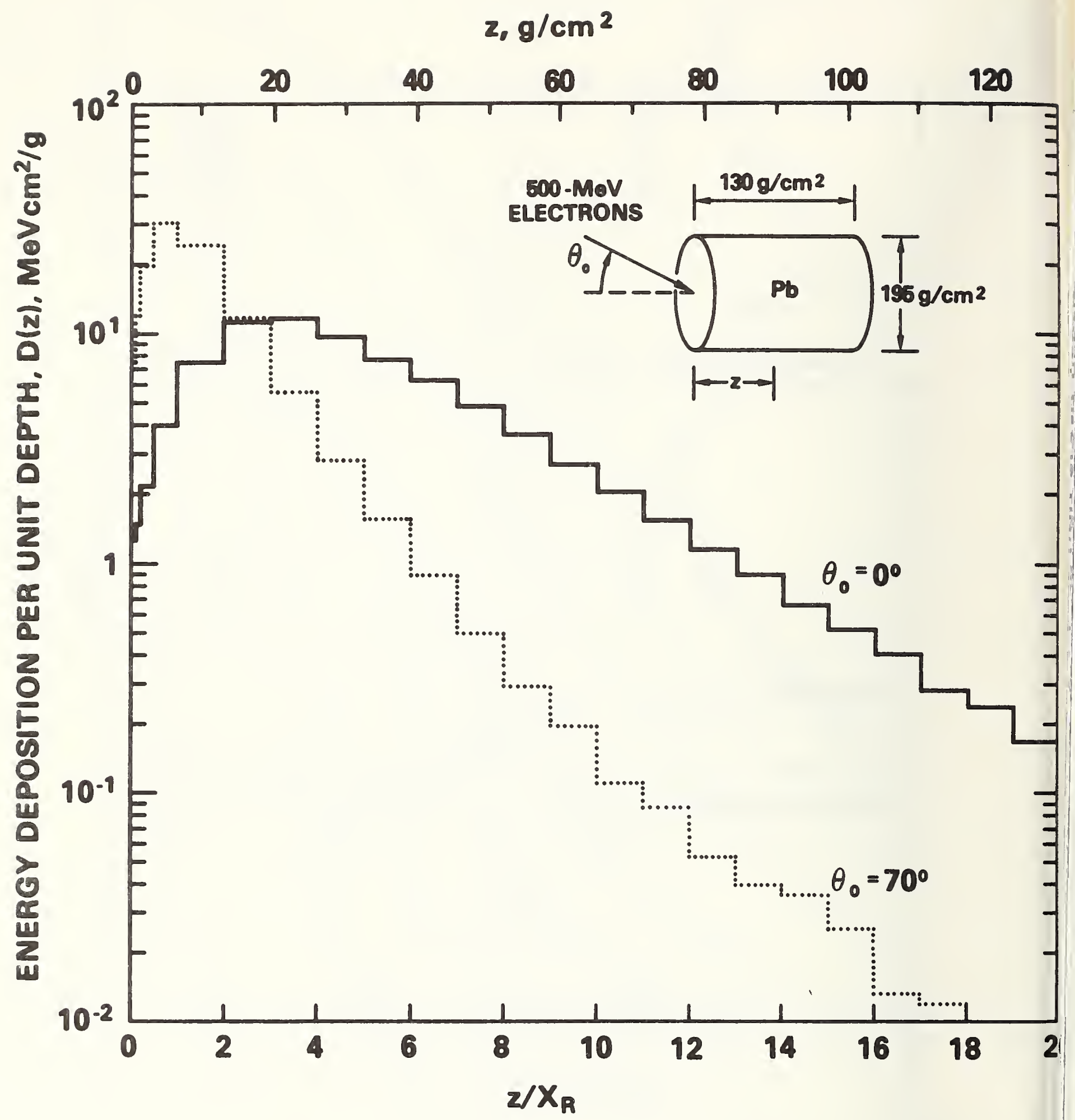

Fig. $8 a$ 
$\mathrm{z} / \cos \theta_{0}, \mathrm{~g} / \mathrm{cm}^{2}$

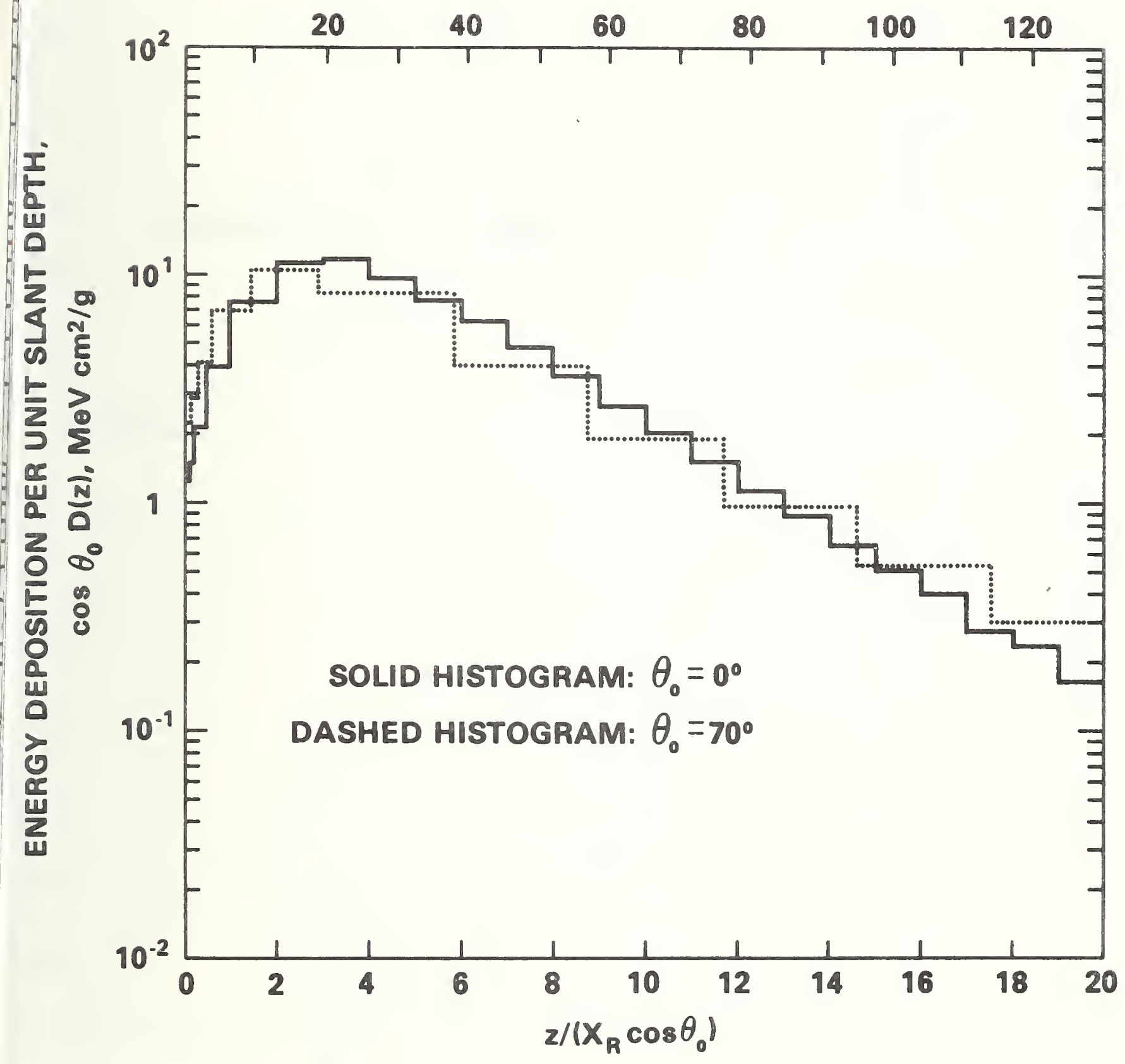

Fig. 8b 

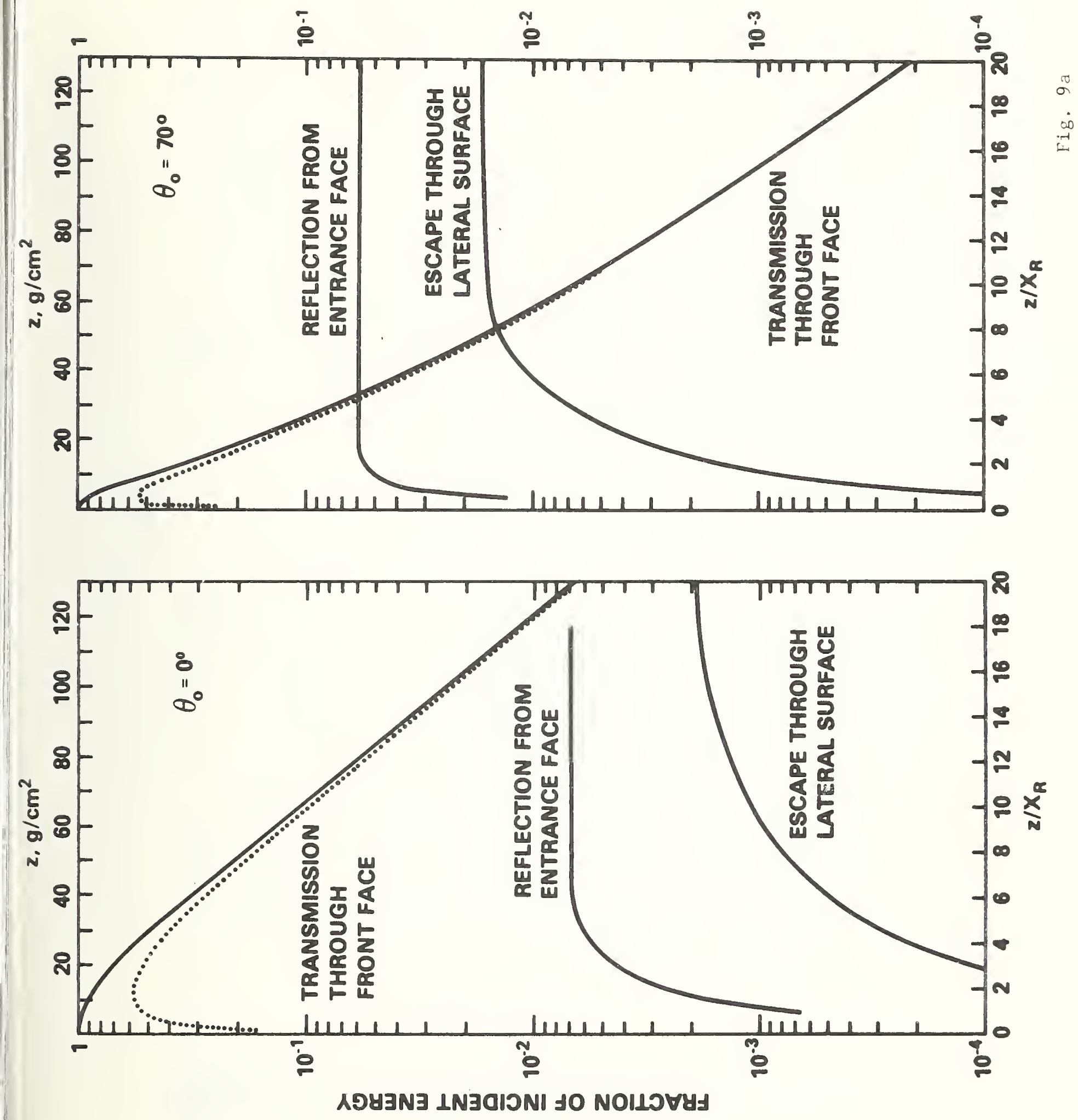


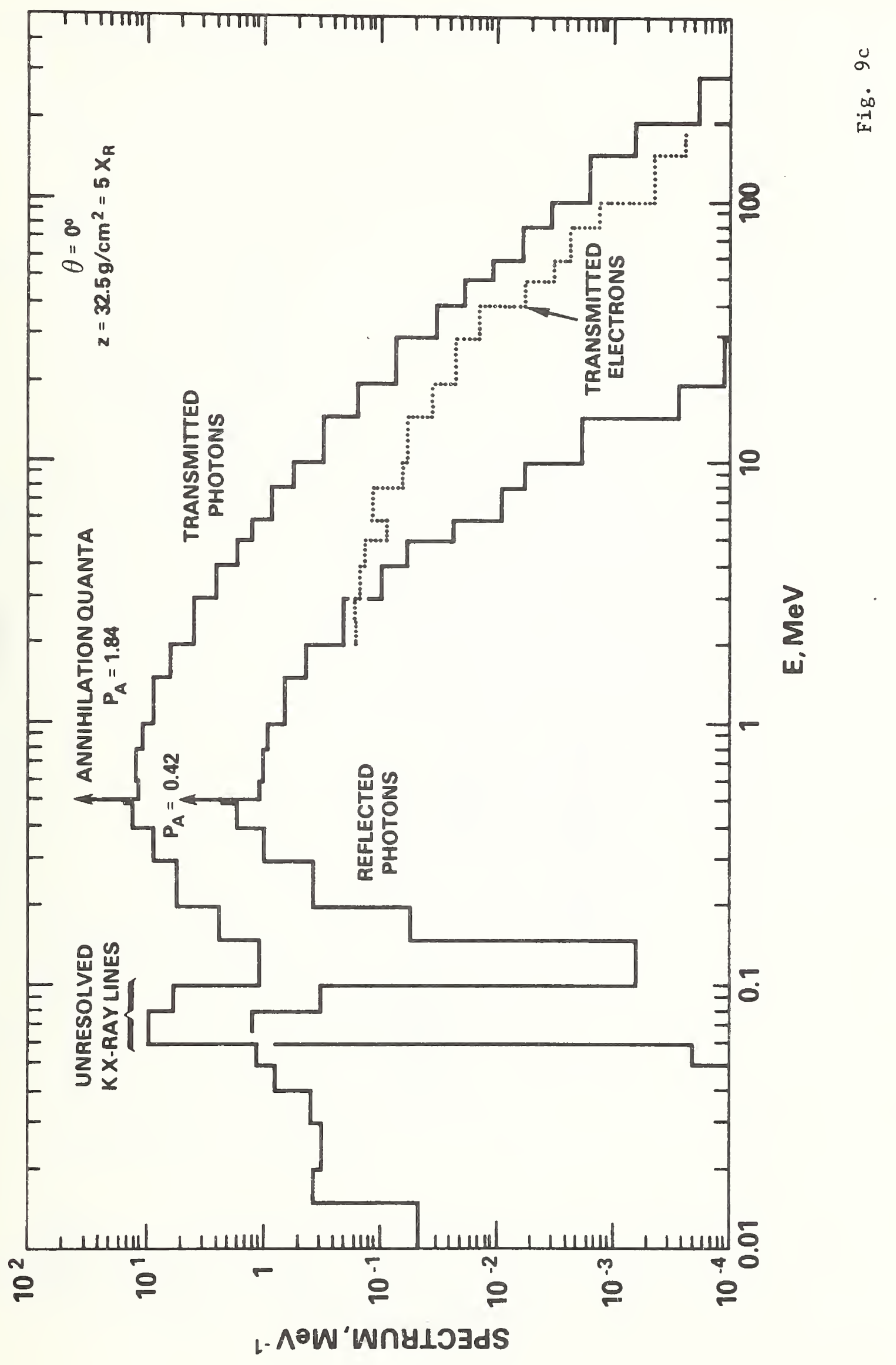




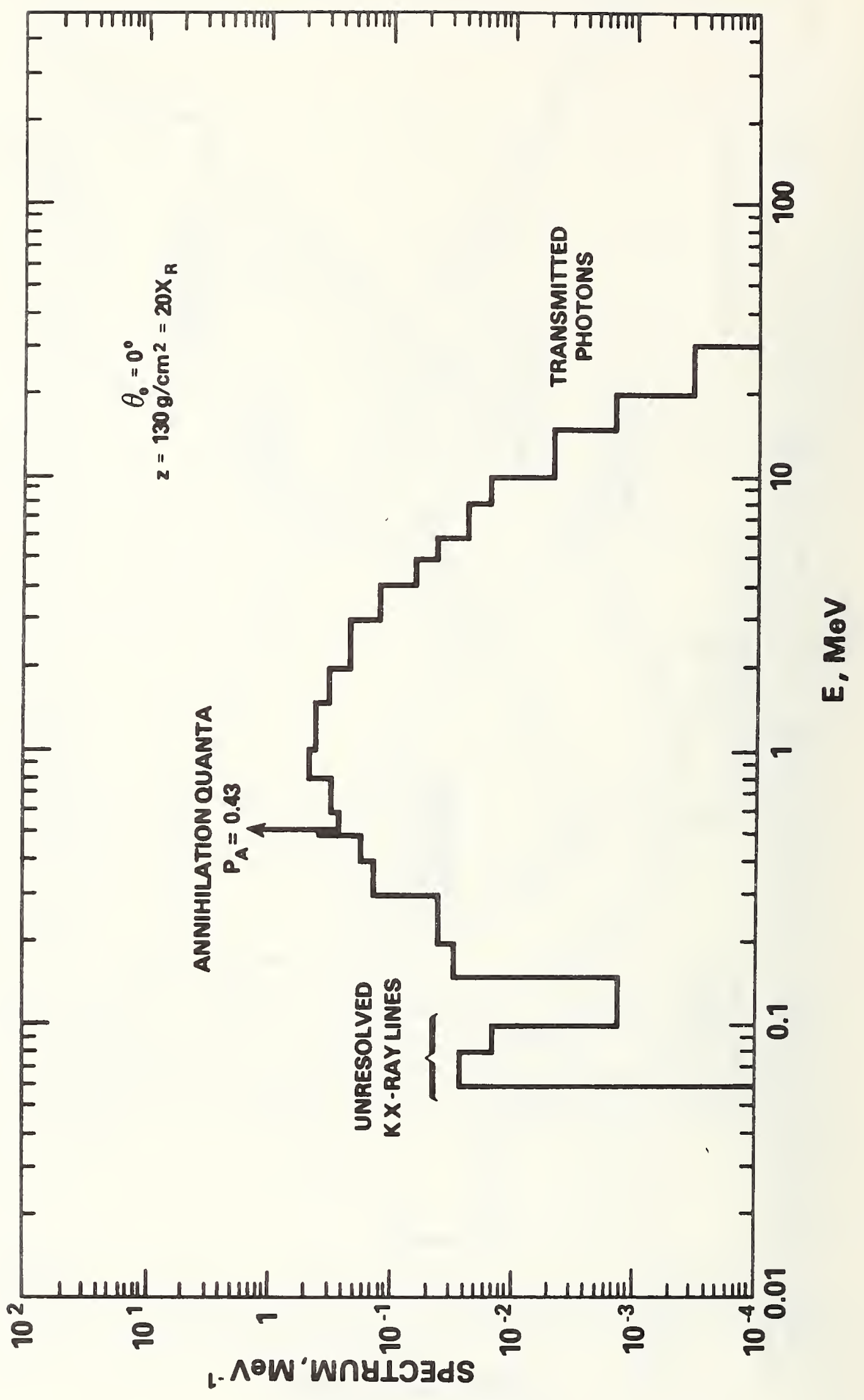




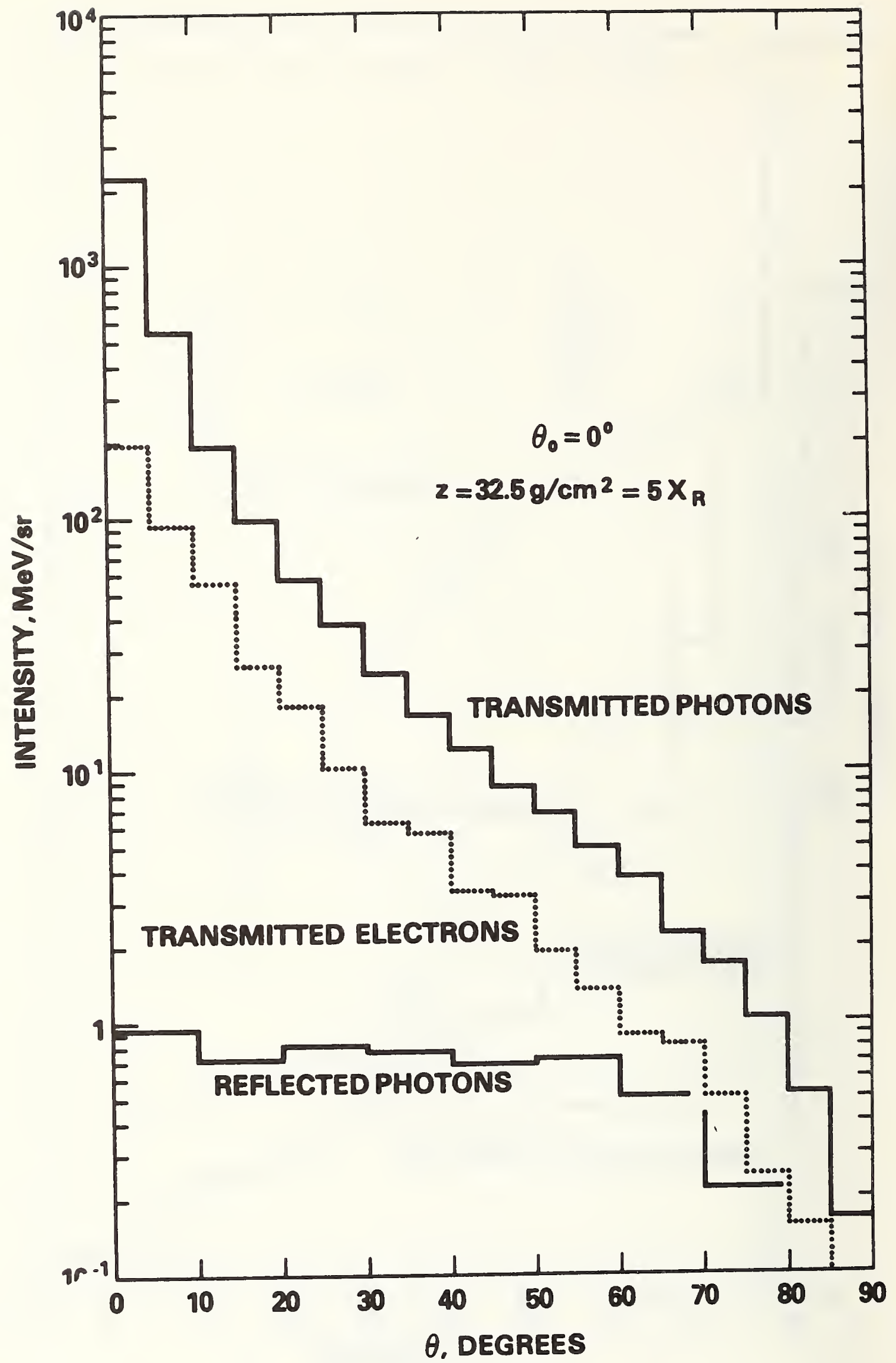

Fig. 9f 


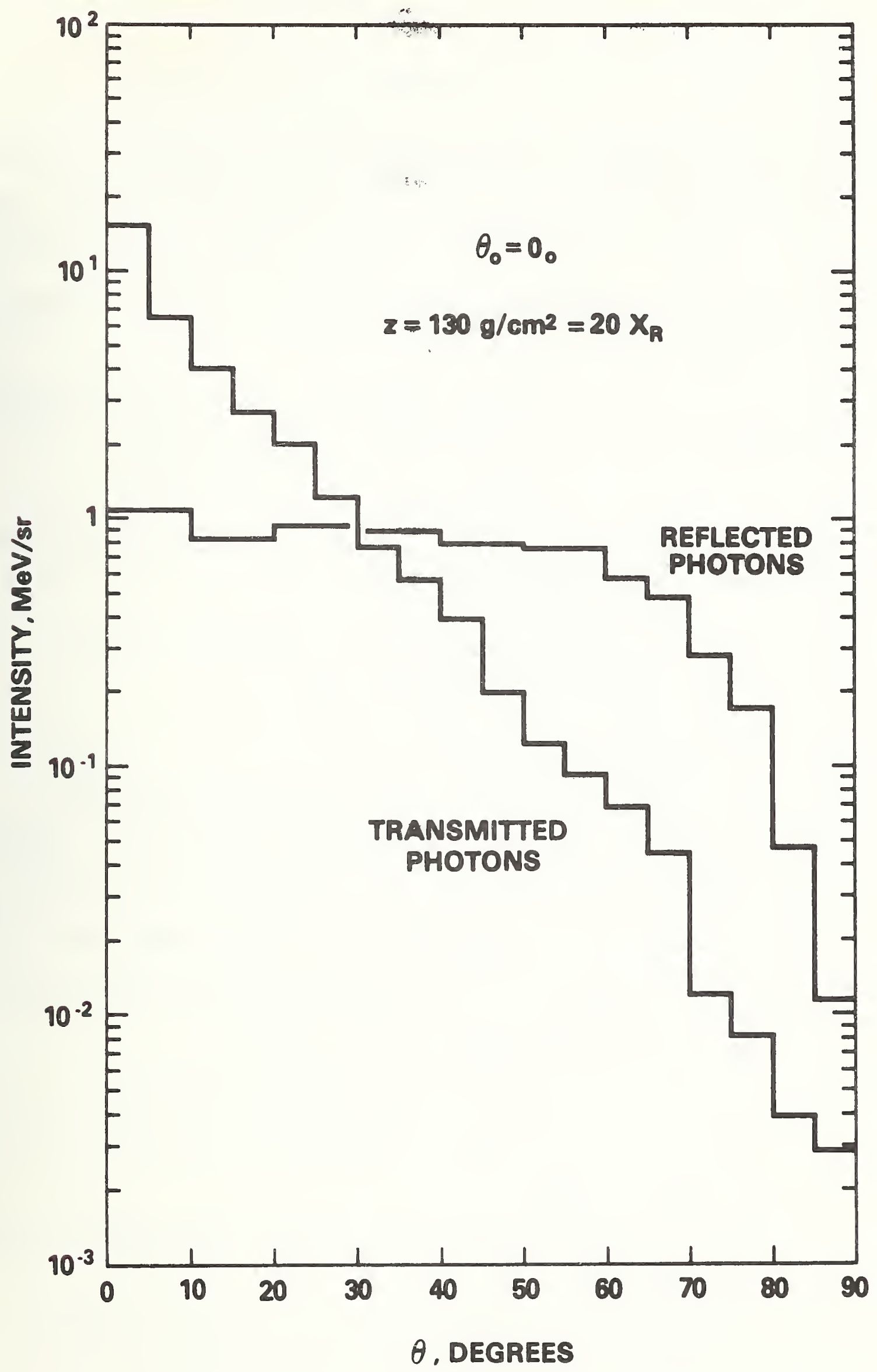

Fig. $9 g$ 


\section{REFERENCES}

[1] M. J. Berger, Methods in Computational Physics, B. Adler, S. Fernback and M. Rotenberg, Eds., Vol. I, p. 135, Academic Press, New York (1963).

[2] S. M. Seltzer, Transmission of Electrons Through Foils, National Bureau of Standards Report NBSIR 74-457 (1974); S. M. Seltzer and M. J. Berger, Nuc1. Instr. Meth. 119, 157 (1974).

[3] M. J. Berger and S. M. Seltzer, Computer Code 107, Radiation Shielding Information Center, Oak Ridge National Laboratory (1968).

[4] M. J. Berger and S. M. Seltzer, Phys. Rev. C 2, 621 (1970).

[5] M. J. Berger and S. M. Seltzer, Ann. New York Acad. Sci. 161, 8 (1969).

[6] L. Landau, J. Phys. USSR 8, 201 (1944).

[7] S. Goudsmit and G. J. L. Saunderson, Phys. Rev. 57, 24 (1940).

[8] N. F. Mott, Proc. Roy. Soc. A124, 425 (1929).

[9] G. Molière, Z. Naturforsch. 3a, 78 (1948).

[10] B. Rossi, High-Energy Particles, Prentice-Hal1, New York (1952).

[11] J. C. Butcher and H. Messel, Nucl. Phys. 20, 15 (1960); D. F. Crawford and H. Messel, Phys. Rev. 128, 2352 (1962); H. Messel, A. D. Smirnov, A. A. Valfolomeev, D. F. Crawford and J. C. Butcher, Nucl. Phys. 39, 1 (1962); H. Messel and D. F. Crawford, Electron-Photon Shower Distribution Function Tables, Pergamon Press, New York (1970).

[12] C. D. Zerby and H. S. Moran, J. Appl. Phys. 34, 2445 (1963).

[13] H. H. Nagel and C. H. Schlier, Z. f. Phys. 174, 464 (1963); H. Nagel, Z. Phys. 186, 319 (1965).

[14] U. Völke1, Deutsches Elektron-Synchrotron Report DESY 65/6, July 1965; DESY 67/16, May 1967.

[15] R. G. Alsmiller and H. S. Moran, Nucl. Sci. Eng. 38, 131 (1969). 
[16] E. Longo and I. Sestili, Nucl. Instr. Methф. 128, 283 (1975).

[17] R. L. Ford and W. R. Nelson, EGS/PEGS, Computer Programs for Monte Carlo Simulation of Electromagnetic Cascade Showers, in preparation.

[18] K. Shin, Y. Hayashida and T. Nakamura, Nuc. Instr. Meth. 151, 271 (1978); T. Nakamura, H. Hirayama and K. Shin, Nucl. Instr. Meth. 151, 277 (1978).

[19] C. J. Crannel1, H. Crannell, R. R. Whitney and H. D. Zeman, Phys. Rev. 184, 426 (1969).

[20] W. R. Nelson, Stanford Linear Accelerator Center, private communication, January 1978.

[21] H. W. Koch and J. W. Motz, Rev. Mod. Phys. 31, 920 (1959).

[22] P. V. Vavilov, Zh. Exper. Teor. Fiz. 32, 320 (1957); JETP $\underline{5}, 749$ (1957). 


\begin{tabular}{|c|c|c|c|}
\hline $\begin{array}{l}\text { U.S. DEPT. OF COMM. } \\
\text { BIBLIOGRAPHIC DATA } \\
\text { SHEET }\end{array}$ & $\begin{array}{l}\text { 1. PUBLICATION OR REPORT NO. } \\
\text { NBSIR } 78-1534\end{array}$ & $\begin{array}{l}\text { 2. Gov't Accession } \\
\text { No. }\end{array}$ & 3. Recipient's Accession No. \\
\hline \multicolumn{3}{|l|}{ 4. TITLE AND SUBTITLE } & 5. Publication Date \\
\hline \multicolumn{3}{|c|}{$\begin{array}{l}\text { Monte Carlo Studies of Electron and Photon Transport } \\
\text { at Energies up to } 1000 \mathrm{MeV} \text {. }\end{array}$} & 6. Performin B Organization Code \\
\hline \multicolumn{3}{|c|}{ 7. AUTHOR(S) Stephen M. Seltzer } & 8. Performing Organ. Report No. \\
\hline \multicolumn{3}{|c|}{ 9. PERF ORMING ORGANIZATION NAME AND ADDRESS } & $\begin{array}{c}\text { 10. Project/Task/Work Unit No. } \\
5331439\end{array}$ \\
\hline \multicolumn{3}{|c|}{$\begin{array}{l}\text { NATIONAL BUREAU OF STANDARDS } \\
\text { DEPARTMENT OF COMMERCE } \\
\text { WASHINGTON, D.C. } 20234\end{array}$} & 11. Contract/Grant No. \\
\hline \multirow{2}{*}{\multicolumn{3}{|c|}{ 12. Sponsoring Organization Name and Complete Address (Street, City, State, ZIP) }} & $\begin{array}{l}\text { 13. Type of Report \& Period } \\
\text { Covered }\end{array}$ \\
\hline & & & 14. Sponsoring Agency Code \\
\hline
\end{tabular}

15. SUPPLEMENTARY NOTES

16. ABSTRACT (A 200 word or less factual summary of most sidnificant information. If document includes a sienificent bibliography or literature survey, mention it here.)

This report describes calculations of electron-photon showers initiated by electron beams with energies from 50 to $1000 \mathrm{MeV}$. Results obtalned with the Monte Carlo program ETRAN are presented for diverse problems including: (1) the spatial distribution of energy deposited in water, air and lead targets; (2) the escape of scattered electrons and secondary bremsstrahlung from air and lead targets; (3) energy-loss straggling of primary electrons in alr; (4) depth-dependent electron spectra (differential tracklength distributions) in air.

17. KEY WORDS (six to twelve entries; alphabetical onder; capitalize only the first letter of the first key word unless a proper name; separated by semicolons)

Electron; electron photon showers; energy deposition; monte carlo method; photon; straggling; transport theory

18. AVAILABILITY Unlimited

For Official Distribution. Do Not Release to NTIS

Order From Sup. of Doc., U.S. Government Printing Office

Washington, D.C. 20402, SD Stock No. SN003-003

Order From National Technical Information Service (NTIS) Springfield, Virginia 22161

\begin{tabular}{|c|c|}
\hline $\begin{array}{c}\text { 19. SECURITY CLASS } \\
\text { (THIS REPURT) } \\
\text { X } \\
\text { UNCL ASSIFIED }\end{array}$ & 21. NO. OF PAGE \\
\hline $\begin{array}{c}\text { 20. SECURITY CLASS } \\
\text { (THIS PAGE) } \\
\text { UNCLASSIFIED }\end{array}$ & 22. Price \\
\hline
\end{tabular}


